\title{
Application of Metamodels to Identification of Metallic Materials Models
}

\author{
Maciej Pietrzyk, Jan Kusiak, Danuta Szeliga, Lukasz Rauch, \\ Lukasz Sztangret, and Grzegorz Górecki
}

AGH University of Science and Technology, Aleja Adama Mickiewicza 30, 30-059 Kraków, Poland

Correspondence should be addressed to Jan Kusiak; kusiak@agh.edu.pl

Received 30 September 2015; Accepted 6 December 2015

Academic Editor: Antonio Riveiro

Copyright (c) 2016 Maciej Pietrzyk et al. This is an open access article distributed under the Creative Commons Attribution License, which permits unrestricted use, distribution, and reproduction in any medium, provided the original work is properly cited.

Improvement of the efficiency of the inverse analysis (IA) for various material tests was the objective of the paper. Flow stress models and microstructure evolution models of various complexity of mathematical formulation were considered. Different types of experiments were performed and the results were used for the identification of models. Sensitivity analysis was performed for all the models and the importance of parameters in these models was evaluated. Metamodels based on artificial neural network were proposed to simulate experiments in the inverse solution. Performed analysis has shown that significant decrease of the computing times could be achieved when metamodels substitute finite element model in the inverse analysis, which is the case in the identification of flow stress models. Application of metamodels gave good results for flow stress models based on closed form equations accounting for an influence of temperature, strain, and strain rate (4 coefficients) and additionally for softening due to recrystallization ( 5 coefficients) and for softening and saturation ( 7 coefficients). Good accuracy and high efficiency of the IA were confirmed. On the contrary, identification of microstructure evolution models, including phase transformation models, did not give noticeable reduction of the computing time.

\section{Introduction}

Continuous progress in numerical modelling of metals processing has been observed during more than half of the century. It became evident that the accuracy of simulations depends mainly on the correctness of the description of boundary conditions and properties of processed materials. The latter problem was the subject of the present work. A number of material models can be found in the scientific literature. Potential extensive predictive capabilities of these models are useful only when proper identification was performed on the basis of experiments. Interpretation of the results of various experiments is the main part of the identification, which usually uses inverse analysis (IA) with finite element (FE) simulation of the test [1-4]. That approach allows elimination of the influence of various disturbances, such as friction or heating due to deformation or due to phase transformations. The published results show that IA improves the accuracy of interpretation of experimental tests significantly.
Various material models used in simulations of thermomechanical processing and various experimental tests performed to identify these models were investigated in the present work. These models describe flow stress, microstructure evolution, and phase transformations. Plastometric tests [4], stress relaxation tests [5], and dilatometric tests [6] are used, respectively, to identify these models. Direct problem model is the model, which is used in the IA to simulate experiment. Thus, finite element (FE) method is used to simulate plastometric tests while JMAK (Johnson-Mehl-AvramiKolmogorov) equation describes microstructure evolution and phase transformations in stress relaxation and dilatometric tests. The direct problem model has to be executed at least once for each calculation of the objective function in the inverse analysis. However, the tests are often performed in various conditions (temperatures, strain rates, and cooling rates) and each calculation of the objective function in the inverse analysis requires several runs of the direct problem model. It means that identification of the models would 
require long computing times. Thus, making the inverse analysis more efficient was the main objective of this work. Application of the metamodel in the inverse analysis was proposed in [7] and the possibility of application of this technique to various experiments was further explored in the present work.

The idea of numerical models substitution with metamodels in the inverse solution has been explored for some time now and solutions for structural mechanics [9], dynamic systems [10], or damage [11] were published. To the authors' knowledge, there are no such solutions for materials models used in processing. Therefore, exploring capabilities of metamodels as substitutions for direct problem models in the inverse analyses of material tests was the particular objective of this work. To reach this goal, various models were analysed from the point of view of their mathematical formulation and physical phenomena, which can be accounted for by the models. Selection of the appropriate metamodel for a considered application was one of the objectives of the work. Various metamodels were tested with respect to accuracy and number of training data and capability of implementation to the optimization procedure in the inverse analysis. The focus in the paper is on flow stress models, but identification of the phase transformation models is addressed briefly, as well, to make the picture of the identification complete.

\section{Models and Metamodels}

Modelling of materials processing requires knowledge of material properties, which depend on many factors like grain size, grain boundaries, dislocation density, stacking fault energy, and so forth. Due to their complexity and scale, accounting for all these factors is difficult. To overcome this problem polycrystals are described by homogenized models, which represent statistically all mentioned microstructural phenomena.

2.1. Flow Stress Models. A large number of flow stress models for metal forming were published in the second half of the XXth century. These models are characterized by various complexity of mathematical formulation and various predictive capabilities. There were several attempts to classify these models; see, for example, [12, 13]; but there is still a lack of convincing hints for a selection of the most appropriate model for a particular application. Analysis of various models inspired the authors to divide the models into three groups: (i) conventional models, (ii) internal variable method (IVM), and (iii) multiscale models.

By conventional model, we understand closed form equations, which describe flow stress as a function of temperature, strain, and strain rate. Introduction of the internal variables instead of strain as independent variables allowed accounting for the inertia of microstructural phenomena. Dislocation density is the most commonly used internal variable and a variety of dislocation density based models were developed following fundamental works of Estrin, Kocks, and Mecking $[14,15]$ and Sandström and Lagneborg [16]. Multiscale models have developed rapidly in the first decade of this century.
Since substitution of these models with metamodels does not seem possible, they are not considered in the present work.

2.1.1. Conventional Models. Conventional models give good results when conditions of deformation are reasonably monotonous and these models are commonly used in simulations of industrial metal forming processes. The first attempt to describe the flow stress as a function of process parameters is attributed to Hollomon, who proposed the power equation describing flow stress relation on strain. To account for the influence of temperature and strain rate, Hollomon equation was extended to the following form:

$$
\sigma_{p}=A \varepsilon^{B} \dot{\varepsilon}^{C} \exp \left(\frac{D}{R T}\right),
$$

where $\sigma_{p}$ is the flow stress, $\varepsilon$ is the effective strain, $\dot{\varepsilon}$ is the effective strain rate, $T$ is the absolute temperature, $R$ is the Universal Gas Constant, and $A, B, C$, and $D$ are coefficients, and the last one is interpreted as activation energy for plastic deformation.

Equation (1) gives good description of the flow stress in the range of temperatures and strain rates corresponding to the high values of the Zener-Hollomon parameter:

$$
Z=\dot{\varepsilon} \exp \left(\frac{Q_{\mathrm{def}}}{R T}\right),
$$

where $Q_{\text {def }}$ is the activation energy for plastic deformation.

At lower $Z$ material softening due to dynamic recrystallization (DRX) becomes important. Beyond $Z$, the stacking fault energy (SFE) is the material parameter, which determines the tendency of this material to dynamic recrystallization. The lower is SFE, the larger strain is needed to launch DRX. Among a number of amendments of (1) to account for softening, correction term proposed by Hensel and Spittel [17] should be mentioned:

$$
\sigma_{p}=A \varepsilon^{B} \dot{\varepsilon}^{C} \exp (-D \widehat{T}) \exp (-E \varepsilon),
$$

where $A-E$ are coefficients and $\widehat{T}$ is the temperature in ${ }^{\circ} \mathrm{C}$.

For larger strains, flows stress calculated from (3) drops to zero or even to negative values, which is not physical and is the main drawback of this model. In real materials, after rapid drop of the flow stress due to DRX, state of saturation is reached and the flow stress remains constant [18]; see curve with round symbols in Figure 1. Auxiliary arrow in this figure shows that the higher is the Zener-Hollomon parameter $Z$ and the higher is the stacking fault energy (SFE), the lower is the tendency of the material to dynamic recrystallization.

Large number of amendments of (1) to account for both softening and saturation were proposed. Equation (3) has several upgrades, which are implemented in commercial FE codes for metal forming. The flow stress model proposed in [2] is based on different idea of accounting for softening and it was selected as the third model in the present work:

$$
\begin{array}{r}
\sigma_{p}=\left[W A \varepsilon^{B} \exp \left(\frac{D}{R T}\right)+(1-W) E \exp \left(\frac{F}{R T}\right)\right] \dot{\varepsilon}^{C}, \\
W=\exp (-G \varepsilon),
\end{array}
$$

where $A-G$ are coefficients. 


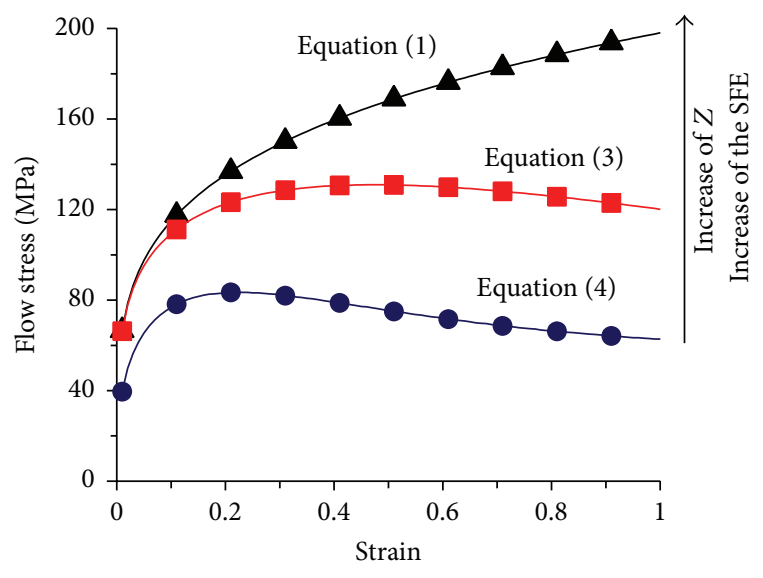

Figure 1: Typical responses of metals subjected to deformation and equations used to describe these responses.

Mathematical form of this model allows the distinguishing of hardening and softening terms; the former is multiplied by $W$ and the latter is multiplied by $(1-W)$. Equations describing the flow stress were further improved to obtain better description of softening and saturation in a wide range of temperatures and strain rates. Typical responses of metals subjected to deformation are shown in Figure 1. Equations (1), (3), and (4) were used in the present work as examples for the demonstration of identification using IA with the metamodel and the equation of [8] was used as a reference model.

2.1.2. Internal Variable Model (IVM). The conventional flow stress model is based on the assumption that stress $\sigma_{p}$ depends on plastic strain $\varepsilon$ and on the current process parameters (e.g., temperature, strain rate), which are grouped in the vector $\mathbf{p}:\left(\sigma_{p}=f(\varepsilon, \mathbf{p})\right)$. The main drawback of this approach is that it does not account for the history of deformation. Whenever the conditions of deformation change, the calculated material response immediately moves to a new equation of state. In real deformation processes, there is some delay in the material response due to microstructural phenomena that occur during deformation. Thus, the internal variable model (IVM) is often used. It allows more accurate description of the material transient behaviour. In this approach, stress is a function of time $t$, again of some process parameters grouped in the vector $\mathbf{p}$, and internal variables, which are grouped in vector $\mathbf{q}:\left(\sigma_{p}=f(\varepsilon, \mathbf{q}, \mathbf{p})\right)$. Usually only one internal variable is considered for metallic materials: the average dislocation density, $\rho$. The evolution of dislocations during deformation is described by

$$
\frac{d \rho(t)}{d t}=\frac{\dot{\varepsilon}}{b l}-A_{2} \rho(t)-\frac{A_{3}}{d} \rho(t) R\left[\rho(t)-\rho_{\mathrm{cr}}\right]
$$

where $\rho(t)$ is the average value of dislocation density, $t$ is the time, $l$ is the mean free path of dislocations, $b$ is the length of the Burgers vector, $d$ is the grain size, and $\rho_{\text {cr }}$ is the critical dislocation density, calculated as a function of the ZenerHollomon parameter. Function $R$ in (5) is calculated as

$$
\begin{aligned}
& R\left[\rho(t)-\rho_{\mathrm{cr}}\right]=0 \quad \text { for } \rho \leq \rho_{\mathrm{cr}}, \\
& R\left[\rho(t)-\rho_{\mathrm{cr}}\right]=\rho\left(t-t_{\mathrm{cr}}\right) \quad \text { for } \rho>\rho_{\mathrm{cr}},
\end{aligned}
$$

where $t_{\mathrm{cr}}$ is time at the beginning of dynamic recrystallization.

Coefficients $A_{2}$ and $A_{3}$ are defined in [19] by Arrhenius law. Flow stress in this model is proportional to the square root of dislocation density according to the formula

$$
\sigma=\sigma_{0}+\alpha b \mu \sqrt{\rho},
$$

where $\sigma_{0}$ is the stress due to elastic deformation, $\mu$ is the shear modulus, and $\alpha$ is constant.

This method is capable of predicting the delay in response due to microstructural processes that take place during deformation, which has been proved experimentally. Details of the IVM solution with one internal variable are described in [19].

\subsection{Microstructure Evolution and Phase Transformation Mod-} els. Changes in microstructure are connected mainly with transformations. JMAK equation was selected to describe kinetics of transformations:

$$
X=1-\exp \left(-k t^{n}\right),
$$

where $X$ is the volume fraction of a new phase and $k, n$ are coefficients.

St3S and DP steel strips were considered in the paper. End of rolling temperature for these steels is about $870^{\circ} \mathrm{C}$; therefore, static recrystallization is the main mechanism which controls microstructure evolution. Equations based on works of Sellars [18], who introduced time for 50\% recrystallization in (8), were used to describe SRX. The microstructure evolution model was not the subject of identification in this paper and is not described.

Phase transformation model is based on (8) as well. Theoretical considerations show that in modelling phase transformations a constant value of coefficient $n$ in (8) can be used. Coefficient $k$ is defined as a temperature function. Modified Gaussian function for parameter $k$ was used in the present paper. Incubation time was calculated from the Arrhenius type equation. Details of this solution are given in [6] and are not repeated here. Equation (8) with different coefficients was used for ferritic, pearlitic, and bainitic transformations. In consequence, the whole model contained 27 coefficients, which had to be identified using inverse analysis.

\subsection{Metamodels}

2.3.1. Development and Evaluation of the Metamodel. The general idea of metamodelling relates to a postulation that metamodel approximates the model of a considered process. Metamodel must correctly correspond to the model and the metamodel output has to be evaluated with a radically lower computing time than using the original model. Thorough discussion of application of metamodelling to optimization 


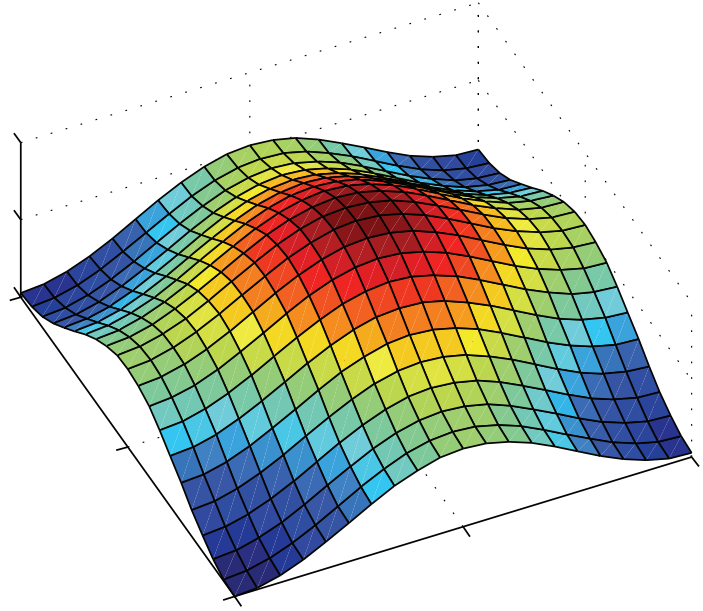

(a)

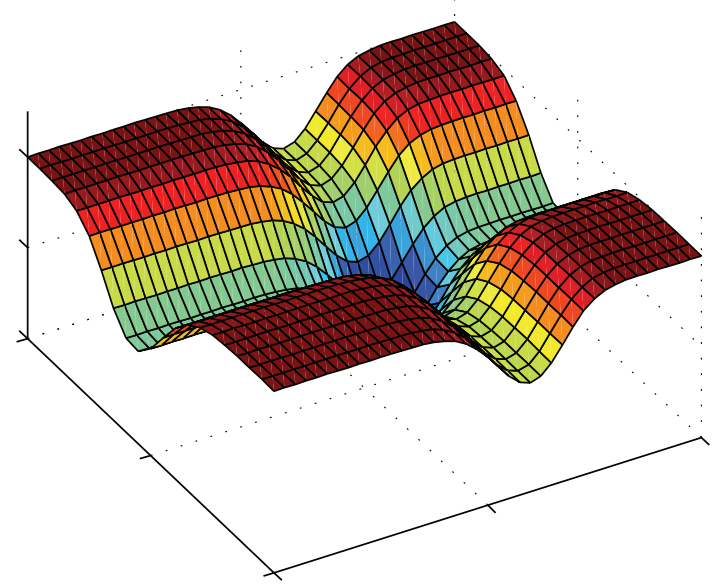

(b)

Figure 2: Rastrigin (a) and Michalewicz (b) benchmark functions.

metal forming processes can be found in [20]. Briefly, metamodelling is a process of construction of an approximation of the analysed model, on the basis of different techniques. In other words, the metamodel is a model of the model. The accuracy of metamodel depends on the used metamodelling technique and on the number of the data points.

One of the objectives of this work was exploring capabilities of various metamodels as direct problem models in inverse analyses of material tests. Three types of tests were investigated; see Section 1. Material models of various complexity of formulation were considered. All of these models are characterized by multimodality [7]. Therefore, simple regression analysis is not effective enough to surrogate them. The Kriging approximation and artificial neural networks (ANN) were used as metamodels. Both techniques are commonly used in the modelling, and their detailed description can be found in numerous textbooks [21-23]. The following aspects were evaluated: accuracy of metamodels depending on a number of training data, metamodels capabilities of use in the optimization procedure, and accuracy of the inverse solution based on the metamodel.

The choice of the metamodelling technique was based on the comparison of the metamodelling results of the following two benchmark functions. The first was Rastrigin test function (Figure 2(a)) given by equation

$$
\begin{aligned}
f\left(x_{1}, x_{2}\right)= & 20+\left[x_{1}^{2}-10 \cos \left(2 \pi x_{1}\right)\right] \\
& +\left[x_{2}^{2}-10 \cos \left(2 \pi x_{1}\right)\right] ; \quad x_{1}, x_{2} \in[0,1],
\end{aligned}
$$

and the second was Michalewicz test function (Figure 2(b)) described by the equation

$$
\begin{aligned}
f\left(x_{1}, x_{2}\right)= & \sin \left(x_{1}\right)\left[\sin \left(\frac{x_{1}^{2}}{\pi}\right)\right]^{20} \\
& -\sin \left(x_{2}\right)\left[\sin \left(\frac{x_{2}^{2}}{\pi}\right)\right]^{20} ; \\
& x_{1} \in[1,3], x_{2} \in[1,2] .
\end{aligned}
$$

The Rastrigin and Michalewicz functions were used mainly to compare metamodelling techniques in terms of accuracy and required memory size. Two different metamodelling techniques were tested: ANN and Kriging. The aim of research was the comparison of the accuracy of these two metamodelling techniques in relation to the number of experimental data points. Since the used benchmark functions were only two-dimensional functions, the metamodelling was performed using 50, 100, and 200 experimental points. The accuracy of metamodels was evaluated using error defined by the following equation:

$$
\Phi=\frac{1}{n} \sqrt{\sum_{i=1}^{n}\left(y_{i}^{m}-y_{i}^{c}\right)^{2}},
$$

where $n$ is the number of testing points and $y^{m}, y^{c}$ are the values measured and calculated from metamodels, respectively.

The obtained results of the ANN and Kriging metamodels of both considered benchmark functions are graphically shown in Figures 3 and 4, respectively, while the metamodels errors are presented in Table 1.

Performed analysis confirms that metamodel error decreases, while a number of experimental points increase (but, on the other hand, the increase of number of experimental data points escalates the research costs). For small number of experimental points, Kriging technique is better in the case of both tested functions. However, when the number of points is higher, ANN technique gives better results for function (9), while Kriging is better in the case of function (10). It is not possible to point out which metamodelling technique is better, based on the accuracy only. Therefore, as a decisive criterion, the memory complexity was taken into account. In the case of ANN-based metamodel, the number of experimental points does not influence the memory size of metamodel. On the contrary, in the case of Kriging metamodel, the memory size of metamodel increases significantly, while the number of experimental points increases. Table 2 presents the required memory size of Kriging metamodel (in bytes) with respect 

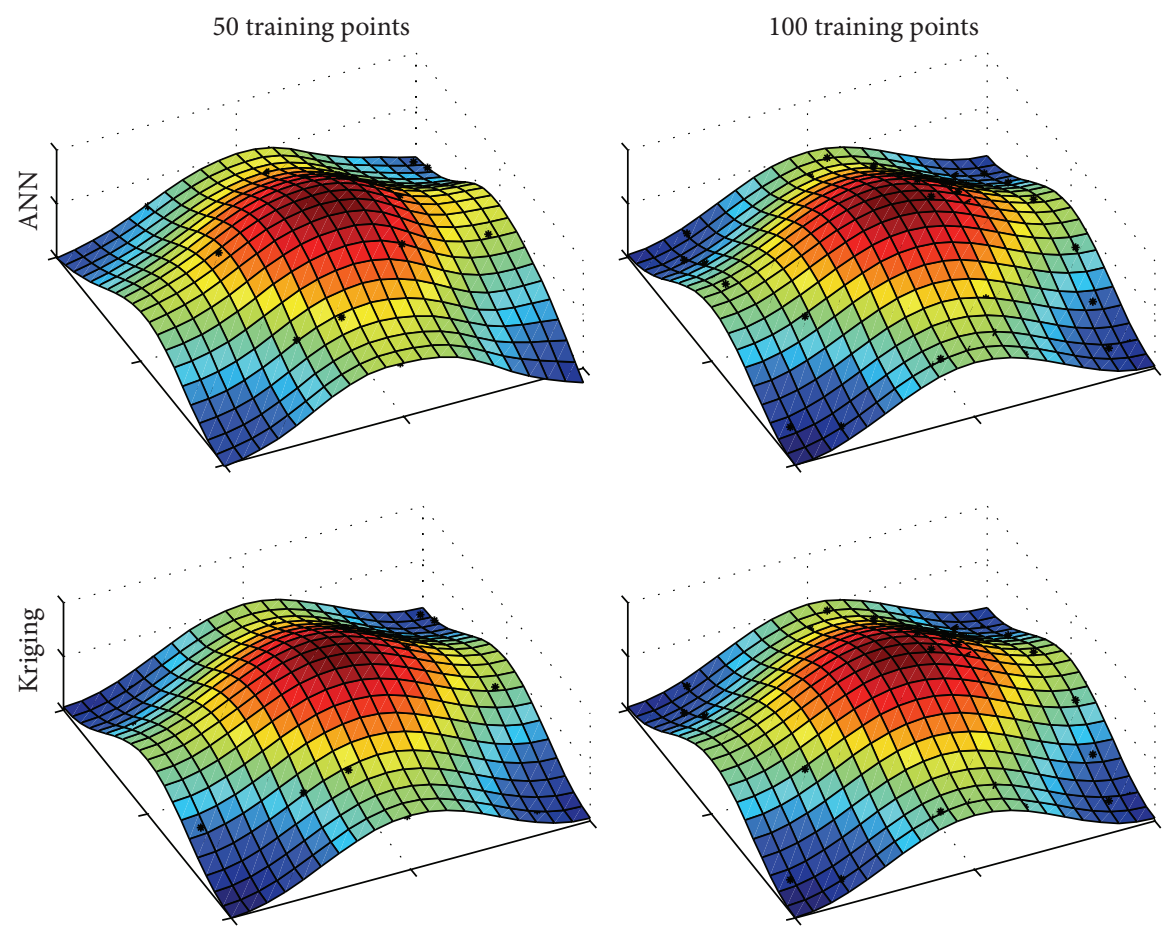

FIGURE 3: Metamodels of the Rastrigin function (9) obtained using the ANN and Kriging methods, based on 50, 100, and 200 experimental points.

50 training points
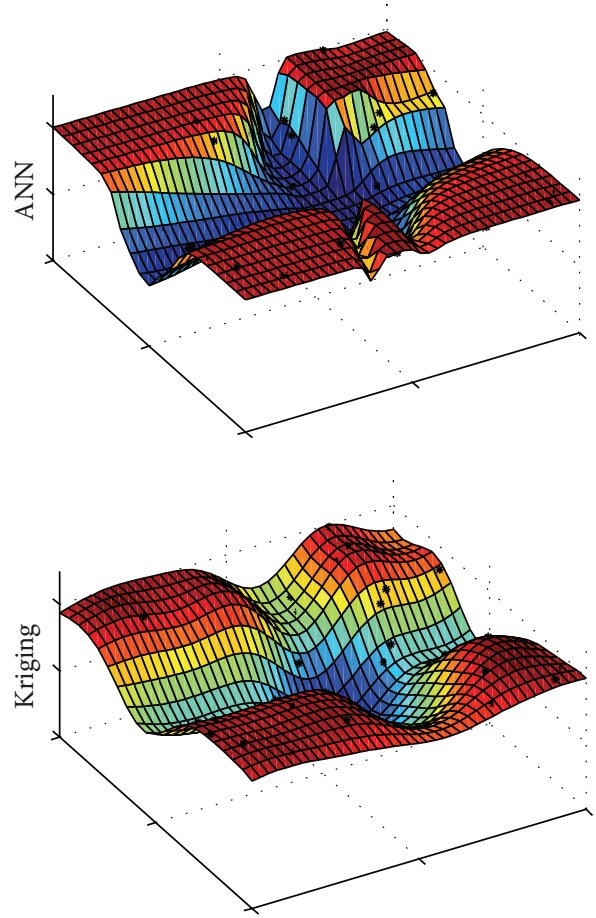

100 training points
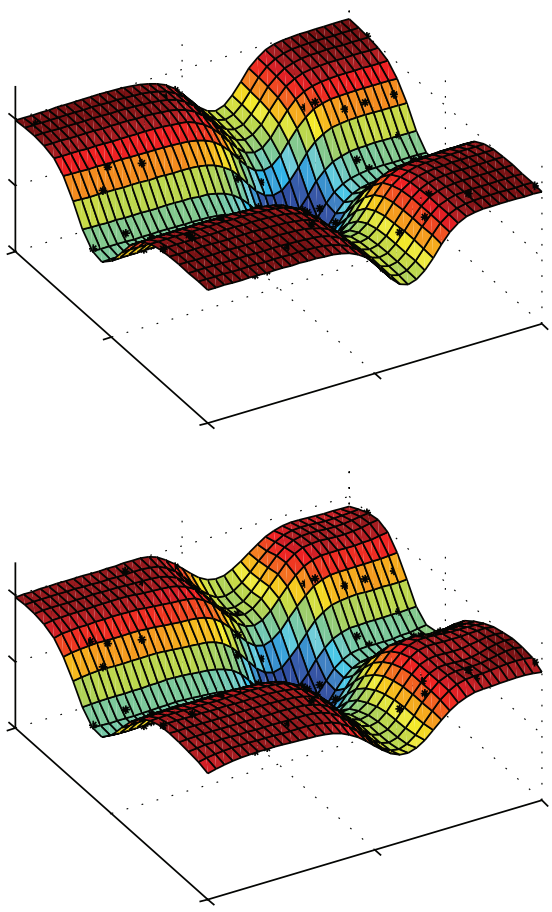
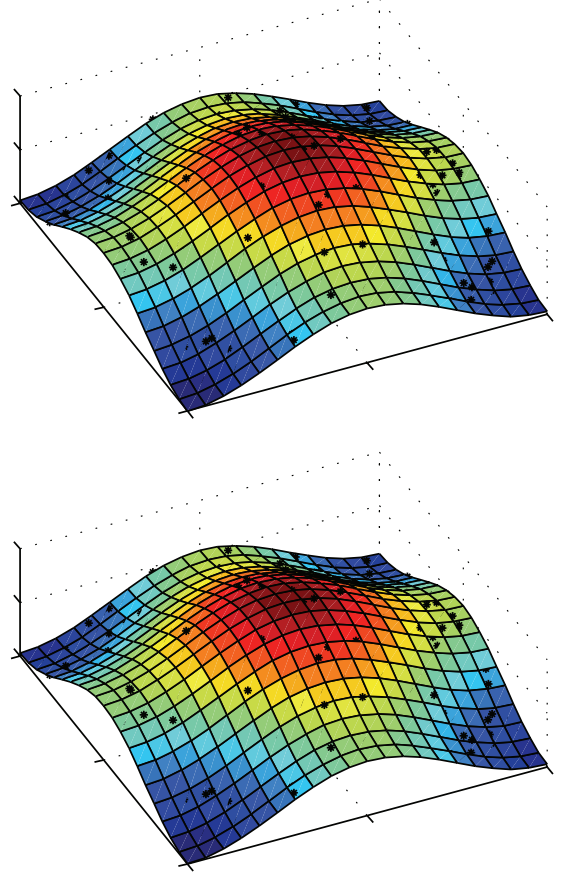

200 training points

FIgURE 4: Metamodels of the Michalewicz function (10) obtained using the ANN and Kriging methods, based on 50, 100, and 200 experimental points. 
TABLE 1: Comparison of the errors of metamodels of benchmark functions (9) and (10).

\begin{tabular}{lccc}
\hline Test function & Number of experimental points & ANN error & Kriging error \\
\hline \multirow{3}{*}{ Rastrigin } & 50 & 0.0446 & $4.32 E-04$ \\
& 100 & 0.0023 & $9.26 E-06$ \\
\hline \multirow{3}{*}{ Michalewicz } & 200 & $1.89 E-04$ & $9.66 E-07$ \\
& 50 & 0.0131 & 0.0082 \\
& 100 & $5.12 E-04$ & 0.0015 \\
& 200 & $2.36 E-05$ & $2.15 E-04$ \\
\hline
\end{tabular}

to the number of experimental points used for training. The required memory size for $\mathrm{ANN}$ metamodels depended on the topology and it did not exceed $20 \mathrm{kB}$.

Since the number of available training points was relatively high (in some cases more than 20000 points, obtained in former authors' research), metamodels used in the present work were built using the ANN technique.

The analysis of the six examples presented in Table 2 shows that the memory size of Kriging metamodel depends squarely on the number of experimental points. Comparison of discussed techniques can also be found in [24] where the metamodelling of laminar cooling of dual-phase steel strips after hot rolling was presented and where the ANN technique gives lower error in metamodelling of real industrial process.

2.3.2. Clusterization of the ANN. Numerical tests showed that the error of the ANN increases with the increasing complexity of the model (increasing number of variables). This error is further magnified in optimization. Therefore, the possibility of improvement of the accuracy of the ANN by constraining the domain of the variables values was explored. The advantage was made from the fact that in the optimization in the inverse analysis the location of the output of the ANN has to be close to the value obtained from measurements. It inspired the authors to apply the clusterization of the ANN on the basis of the value of the output. The schematic illustration of this approach for only two optimization variables is shown in Figure 5. Two types of networks were used. The first was a general network trained for all available data in the whole domain of values of variables. This network was used at the primary stage of the optimization, when the solution is far from the optimum. At the final stage of the optimization, the ANN output is known, because it has to be close to the measured value, for example, force $F_{m}$. Therefore, the whole domain of outputs was divided into intervals and separate network was trained for each interval. The accuracy of these networks, which are called clusterized neural networks (CANN), was much better than that of the global network.

\section{Experiments}

St3S and DP600 steels with the chemical compositions in Table 3 and $\mathrm{CuCr}$ alloy with the chemical composition in Table 4 were selected as the main investigated materials; however, more materials tested in other projects were subject
TABLE 2: Memory size of Kriging metamodel with respect to the number of experimental points.

\begin{tabular}{lc}
\hline $\begin{array}{l}\text { Number of } \\
\text { experimental points }\end{array}$ & $\begin{array}{c}\text { Memory size of Kriging } \\
\text { metamodel [B] }\end{array}$ \\
\hline 100 & 87064 \\
200 & 331864 \\
500 & 2026264 \\
1000 & 8050264 \\
2000 & 32098264 \\
4000 & 128194264 \\
\hline
\end{tabular}

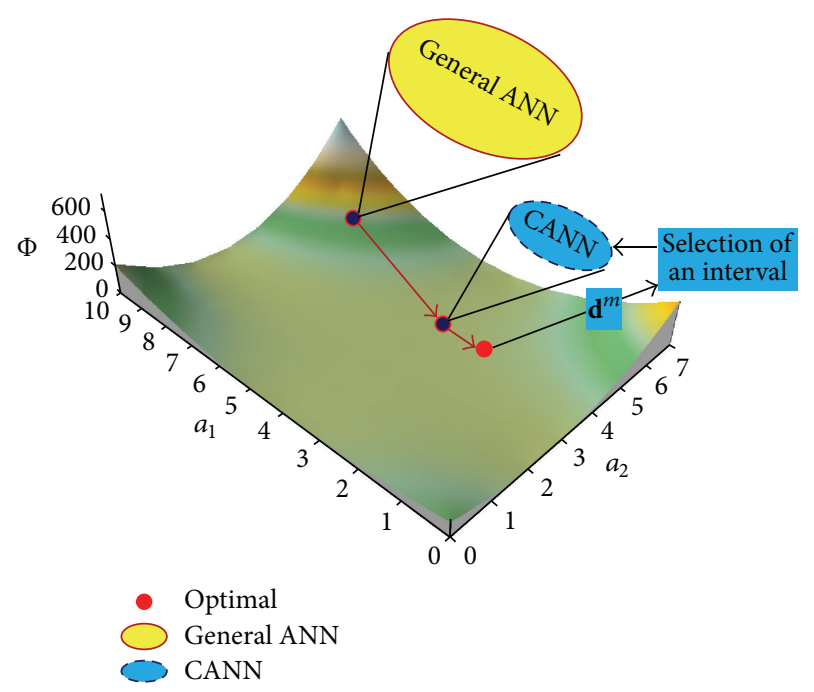

Figure 5: The idea of application of clusterization of the ANN during optimization.

to the inverse analysis, as well. All the tests were performed with Gleeble 3800 simulator and dilatometer DIL805.

3.1. Compression Tests. In general, the stress versus strain, strain rate, temperature, and so forth (depending on conditions) relation is determined on the basis of the results of experiments, which are called plastometric tests. The tests can have various forms (tension, compression, and torsion) depending on further use of the flow curve. Advantages and disadvantages of plane strain compression (PSC), cylinder (UC), and ring (RC) compression tests as well as torsion tests (TT) are discussed in the literature; see, for example, [25]. On 
TABLE 3: Chemical composition of the investigated steels St3S and DP600, wt\%.

\begin{tabular}{lccccccccrrr}
\hline Steel & $\mathrm{C}$ & $\mathrm{Mn}$ & $\mathrm{Si}$ & $\mathrm{Cr}$ & $\mathrm{Cu}$ & $\mathrm{P}$ & $\mathrm{S}$ & $\mathrm{Ti}$ & $\mathrm{V}$ & $\mathrm{Al}$ & $\mathrm{N}$ \\
\hline St3S & 0.16 & 0.43 & 0.23 & 0.01 & 0.03 & 0.006 & 0.015 & - & - & 0.03 & 0.004 \\
\hline DP600 & 0.071 & 1.45 & 0.25 & 0.55 & 0.02 & 0.01 & 0.006 & 0.002 & 0.005 & 0.022 & 0.0039 \\
\hline
\end{tabular}

TABLE 4: Chemical composition of the investigated $\mathrm{CuCr}$ alloy, wt $\%$.

\begin{tabular}{lcccccc}
\hline $\mathrm{Cr}$ & $\mathrm{Ni}$ & $\mathrm{Si}$ & $\mathrm{Fe}$ & $\mathrm{As}$ & $\mathrm{Bi}$ & $\mathrm{Cu}$ \\
\hline 0.81 & $<0.001$ & $<0.001$ & 0.026 & $<0.001$ & $<5 \mathrm{ppm}$ & Balance \\
\hline
\end{tabular}

the basis of the analysis of various aspects of the tests, UC, PSC, and RC tests which allow large deformation and are easy to perform were selected for further analysis in the present work. Illustration of these tests is shown in Figure 6.

In hot compression, UC samples measuring $\phi 10 \times 12 \mathrm{~mm}$, $\mathrm{RC}$ samples measuring $\phi 7 \times \phi 3.5 \times 4.67 \mathrm{~mm}$, and PSC samples measuring $10 \times 15 \times 20 \mathrm{~mm}$ were used. The tests were performed in a wide range of temperatures of hot deformation $\left(800^{\circ} \mathrm{C}-1230^{\circ} \mathrm{C}\right)$ and cold deformation $\left(20^{\circ} \mathrm{C}-300^{\circ} \mathrm{C}\right)$. Strain rates varied in the range of $0.1 \mathrm{~s}^{-1}-30 \mathrm{~s}^{-1}$ characteristic for industrial forming processes. The procedure for hot tests was composed of preheating at maximum temperature $\left(1230^{\circ} \mathrm{C}\right)$ for $300 \mathrm{~s}$, cooling to the test temperature, remaining at this temperature for 10 seconds, and compression with a total homogeneous strain of 1 .

Samples measuring $\phi 7.5 \times 9 \mathrm{~mm}$ were used in cold compression. The procedure was composed of heating to the test temperature, remaining at this temperature for 10 seconds, and compression with a total strain of 0.8 . Forces recorded during the tests are shown in Figure 7 for the UC and RC tests (DP and St3S steels) and in Figure 8 for the UC and PSC tests (CuCr alloy).

In the conventional interpretation of the tests, the flow stress is calculated as force-to-contact area ratio. The strain is calculated as $(2 / \sqrt{3}) \ln \left(h_{0} / h\right)$ for the PSC test and $\ln \left(h_{0} / h\right)$ for the UC and RC tests $\left(h_{0}, h\right.$ : initial and current height of the sample, resp.). This interpretation assumes uniform strain and stress distribution, which leads to erroneous results. More accurate results giving the same flow stress from various tests are obtained when IA is applied. Validation of the inverse approach with the metamodel for materials in Tables 3 and 4 and for various tests was performed.

3.2. Stress Relaxation Tests and Dilatometric Tests. Stress relaxation tests were performed on Gleeble 3800 simulator. The idea of this test is described in [5]. The results of these tests were used to determine kinetics of static recrystallization and to identify coefficients in microstructure evolution model. Dilatometric tests were performed according to the Standard Practice published by ASTM International: A103304 . Sample dimensions were $\phi 2 \times 4 \mathrm{~mm}$. The technique of tangent to dilatometric curve at the beginning and the end of transformation was used. In the case of transformation occurring one after another, numerical differentiation of the curve was applied. To determine end temperatures for ferritic and pearlitic transformations, the method based on linear transformation of dilatometric curve was applied. All

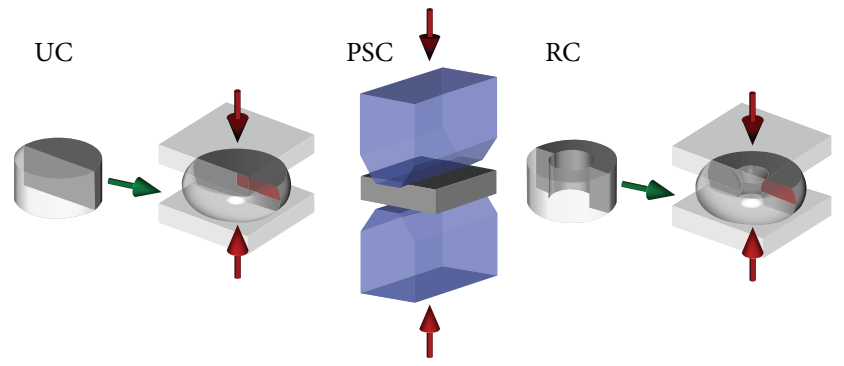

FIgURE 6: Schematic illustration of the compression tests investigated in the present work.

samples were subjected to microstructure analysis using optical microscope. Vickers hardness with the load of $98 \mathrm{~N}$ was measured for each sample.

\section{Sensitivity Analysis and Inverse Analysis}

Identification of models using inverse analysis is usually preceded by the sensitivity analysis. Both of these procedures are described briefly in this chapter.

\subsection{Sensitivity Analysis (SA)}

4.1.1. Objectives of the Sensitivity Analysis and Selection of the SA Method. Sensitivity analysis (SA) allows us to assess the accuracy of the model of the analysed system or process, determine the parameters which contribute the most to the output variability, indicate the parameters which are insignificant and may be eliminated from the model, evaluate these parameters which interact with each other, and determine the input parameters region for subsequent calibration space [26]. The steps of the SA are as follows:

(i) sensitivity measure: the measure expresses the model solution (model output) changes to the model parameter variation;

(ii) selection of the parameter domain: design of experiment technique was used to select the lower number of points, which guaranteed the searching of the whole domain;

(iii) sensitivity calculation: the sensitivities were estimated by Morris OAT (One At a Time) Design method [27], which belongs to the global SA methods.

The information obtained from sensitivity analysis was applied to the inverse method:

(i) To verify whether the objective function is well defined, SA gives information if the sensitivity of the objective function to the parameter changes is large enough to allow estimation of this parameter. 


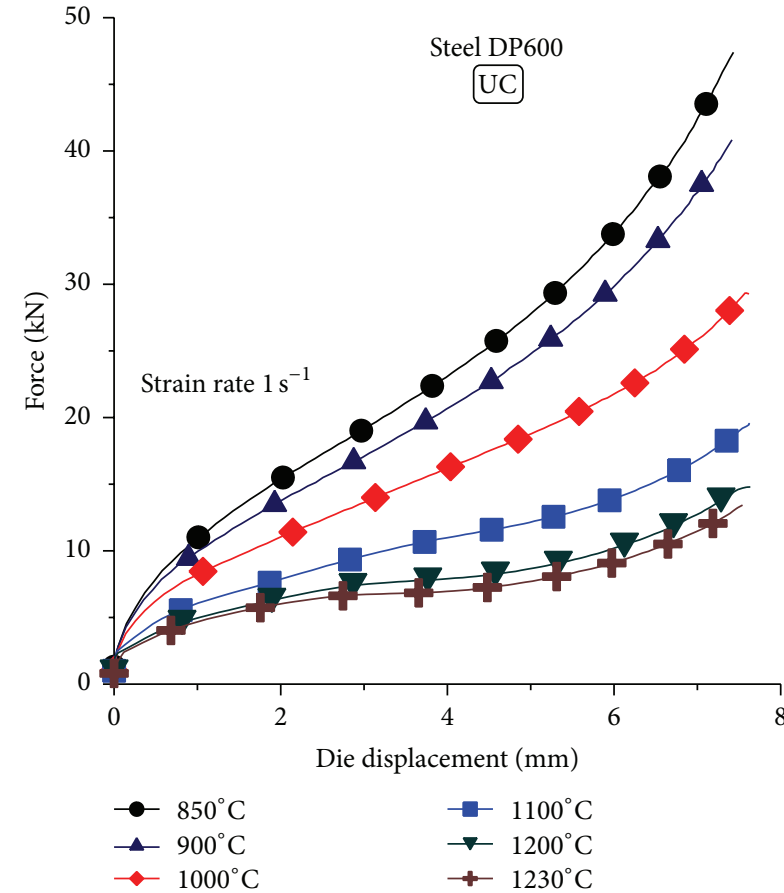

(a)

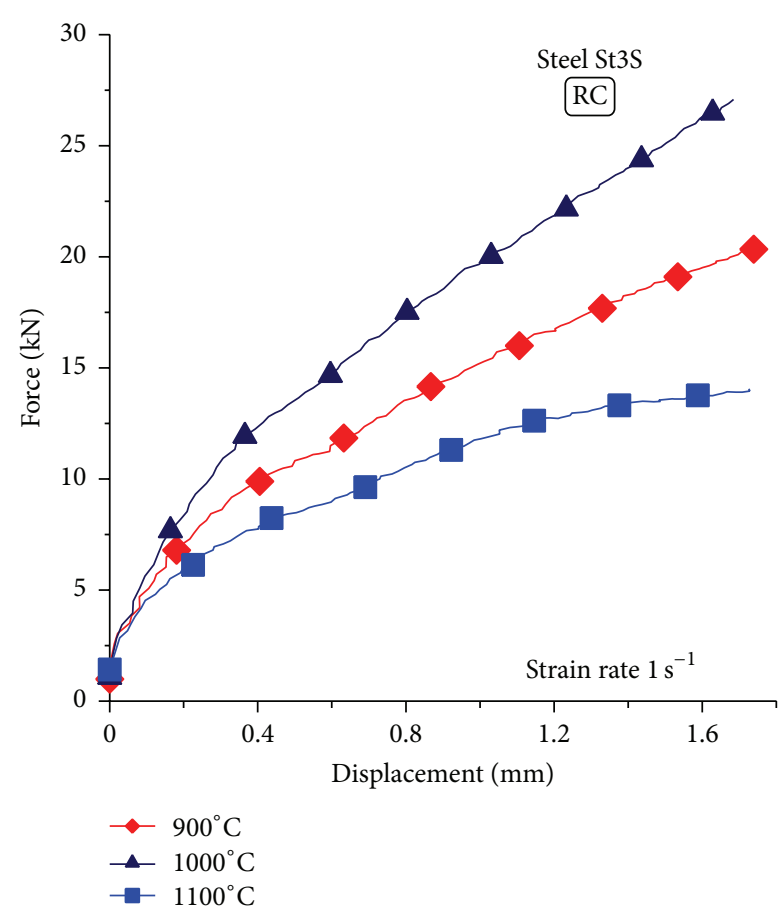

(b)

FIGURE 7: Loads measured in the UC tests for the DP600 steel, strain rate $1 \mathrm{~s}^{-1}$ (a), and for the RC tests for the St3S steel, strain rate $1 \mathrm{~s}^{-1}$ (b).

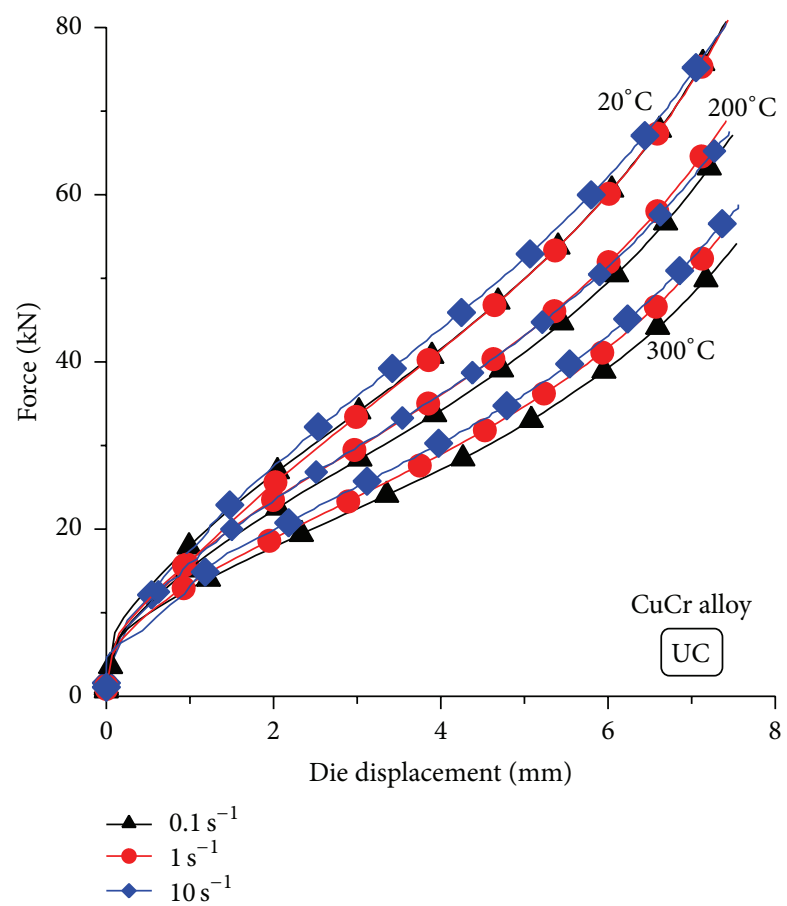

(a)

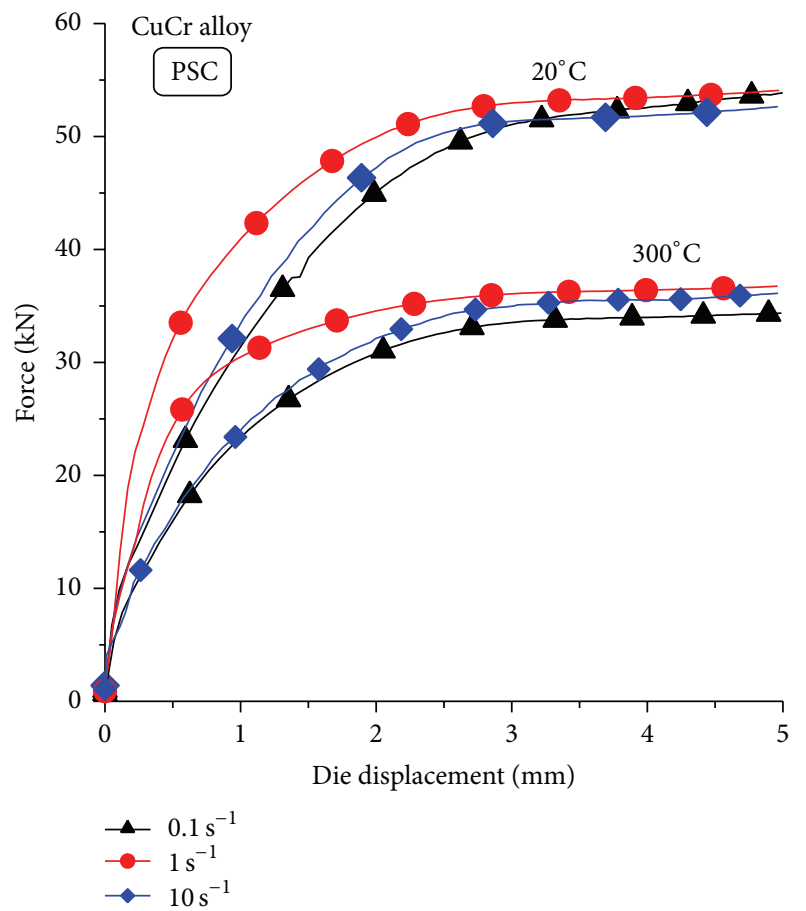

(b)

FIGURE 8: Loads measured in the UC tests (a) and the PSC tests (b) for the CuCr alloy. 
(ii) A preliminary step is to select the starting point or the first population for optimization.

(iii) In optimization process, we construct the hybrid algorithms (the combination of two or more methods) or modified algorithms to increase the procedure efficiency.

Selected Morris OAT Design method [27] is classified as a screening method. The objective was pointing out these parameters of the model, which have influence on the model output. By the parameter, we understand here both model coefficients and process parameters. The screening methods evaluate the statistical importance of the considered parameter and they allow for qualitative assessment of the influence of this parameter on the output. The general idea is the calculation of the sensitivity coefficient $\xi_{i}$ (elementary effect) with respect to the selected parameter $i$ for various values of the remaining parameters. This elementary effect $\xi_{i}$ is calculated as

$$
\xi_{i}(\mathbf{x})=\frac{y\left(x_{1}, \ldots, x_{i-1}, x_{i}+\Delta_{i}, x_{i+1}, \ldots, x_{k}\right)-y(\mathbf{x})}{\Delta_{i}},
$$

where $y$ is the model output, $\mathbf{x} \in \Omega \subset P^{k}$ is the $k$-dimensional vector of model parameters $x_{i}, \Omega$ is technologically allowed domain of parameters $x_{i}$, and $\Delta_{i}$ is the $i$ th parameter disturbance.

The elementary effects $\xi_{i}$ were calculated for randomly selected values of parameters in the whole search domain (screening). The average value of the sensitivity $\xi$ and standard deviation were calculated next. The latter represents degree of nonlinearity of the influence of the selected parameter. SA algorithm proposed by Szeliga [28] was used and the selected results of the analysis are presented below.

4.1.2. Sensitivity of the Flow Stress with respect to the Coefficients in the Model. Since the investigated models are based on closed form equations (1), (3), and (4), the results were consistent. In cold forming, sensitivity to temperature and strain rate is negligible. The results of the sensitivity calculations for (3) and (4) are shown in Figure 9. In hot forming, the flow stress is sensitive to all coefficients, but sensitivity to coefficients responsible for the influence of temperature and for the softening during deformation, as well as sensitivity to temperature and strain rate itself, is particularly large.

4.1.3. Sensitivity of the Phase Transformation Model. Due to complex mutual influence of phase transformations, sensitivity analysis for the phase transformation model is a difficult problem. Changes of kinetics of one transformation (e.g., ferritic) may result in the occurrence or nonoccurrence of another one (e.g., bainitic). Therefore, the problem of the sensitivity analysis of the phase transformation was a subject of the separate work [28] and details are not given here. On the basis of the SA, the number of coefficients in the phase transformations model was decreased to 23 .
4.1.4. Sensitivity of the Inverse Solution with respect to the Process Parameters. Substitution of the FE model with the metamodel was the objective of the present work. To be efficient, once trained, the metamodel should be used for identification of various materials, without additional training. This method will be robust and accurate when the number of input parameters to the metamodel is reasonably low. Beyond coefficients in the flow stress model, there are additional parameters, which have to be considered as model inputs:

(i) temperature and strain rate,

(ii) friction coefficient,

(iii) sample dimensions,

(iv) thermophysical properties of the material, which influence simulation of the temperature.

These problems were investigated in earlier publication [29]. It was shown that since the tests are performed in the furnace and heat exchange with the surrounding and the tool is marginal the sensitivity of the result of the inverse analysis to thermophysical properties is negligible and they were eliminated from the input parameters of the metamodel. Sensitivity to friction was investigated in [29], and although small but still important, sensitivity was observed. See also [28] for more detailed analysis. Therefore, friction coefficient remained as the input parameter.

In consequence, the following parameters were introduced as an input to the metamodel: coefficients in the flow stress equations $(A-G)$, temperature, strain rate, and friction coefficient. Separate metamodel was developed for each standardized sample dimension.

4.2. Inverse Analysis (IA). The inverse algorithm proposed in [4] and validated in [29] was used. A mathematical model of an arbitrary process or physical phenomenon can be described by a set of equations:

$$
\mathbf{d}=F(\mathbf{a}, \mathbf{p}), \quad F: R^{k+l} \longrightarrow R^{r},
$$

where $\mathbf{d}=\left\{d_{1}, \ldots, d_{r}\right\}$ is the vector of outputs of the model (forces, transformation temperatures, and shape of the sample after the tests), $\mathbf{a}=\left\{a_{1}, \ldots, a_{k}\right\}$ is the vector of coefficients of the model, and $\mathbf{p}=\left\{p_{1}, \ldots, p_{l}\right\}$ is the vector of the known process parameters (temperature, strain rate, and cooling rate).

When vectors $\mathbf{p}$ and a are known, the solution of problem (13) is called a direct solution. Inverse solution of problem (13) called identification is defined as the determination of the components of vector a for known vectors $\mathbf{d}$ (from measurements) and $\mathbf{p}$ :

$$
\mathbf{a}=F^{-1}(\mathbf{d}, \mathbf{p}), \quad F^{-1}: R^{r+l} \longrightarrow R^{k} .
$$

When the problem is linear, the inverse function can be usually found and the problem can be solved analytically. In the investigated problem of materials processing, this relation is nonlinear and the problem is transformed into the optimization task. Thus, the objective of the inverse analysis is 


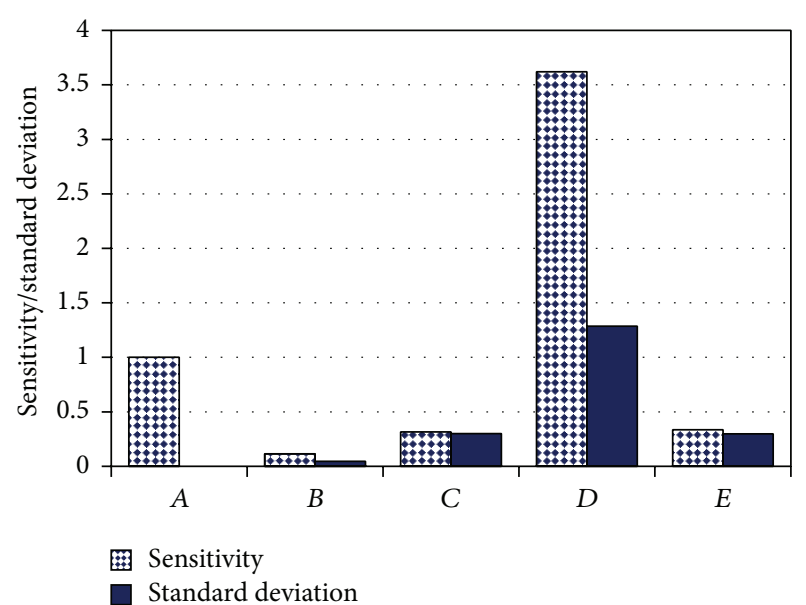

(a)

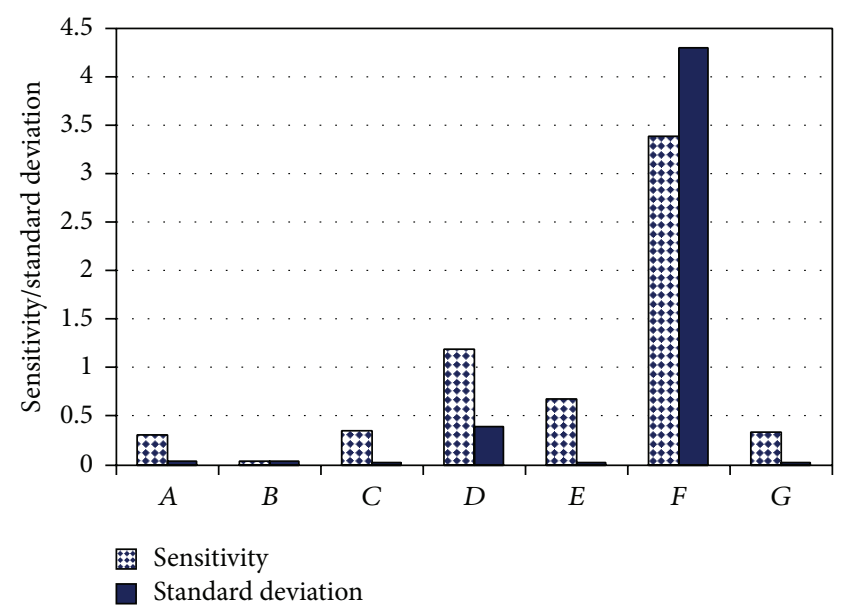

(b)

FIGURE 9: Sensitivity of the flow stress with respect to coefficients in (3) (a) and (4) (b) and standard deviations for these sensitivities.

the determination of the optimum components of vector a by searching for the minimum, with respect to the components of this vector, of the objective function defined as a square root error between measured and calculated components of the vector $\mathbf{d}$ :

$$
\Phi(\mathbf{a}, \mathbf{p})=\sum_{i=1}^{n} \beta_{i}\left[\mathbf{d}_{i}^{c}\left(\mathbf{a}, \mathbf{p}_{i}\right)-\mathbf{d}_{i}^{m}\right]^{2},
$$

where $\mathbf{d}_{i}^{m}$ is the vector with output measured parameters, $\mathbf{d}_{i}^{c}$ is the vector with these parameters calculated by the model, $\beta_{i}$ are weights of the points $(i=1, \ldots, n)$, and $n$ is the number of measurements. Weights in (15) are selected arbitrarily by the user; for example, larger weights can be selected for characteristic points like peak strain.

Thus, inverse analysis is composed of three steps: experiment, FE simulation of the experiment, and optimization. Flow chart of this algorithm is shown with the solid line in Figure 10. To decrease the computing times, FE model was substituted by the metamodel based on the ANN (broken line in Figure 10). After this substitution, various optimization techniques could be used to find the minimum of function (15), even those which require large number of calculations of the objective function. Usually, methods based on the observations of the nature (e.g., genetic algorithms, evolutionary algorithms, immune systems, and aunt algorithm) are used by the authors, because they increase the probability of finding the global minimum.

\section{Results}

Metamodels were developed for all investigated experiments and for all material models. These metamodels were used in identification of material models and the results were compared with the classical inverse solution based on the FE direct problem model.

5.1. Identification of Various Flow Stress Models. The objective function (15) for the inverse analysis of plastometric tests

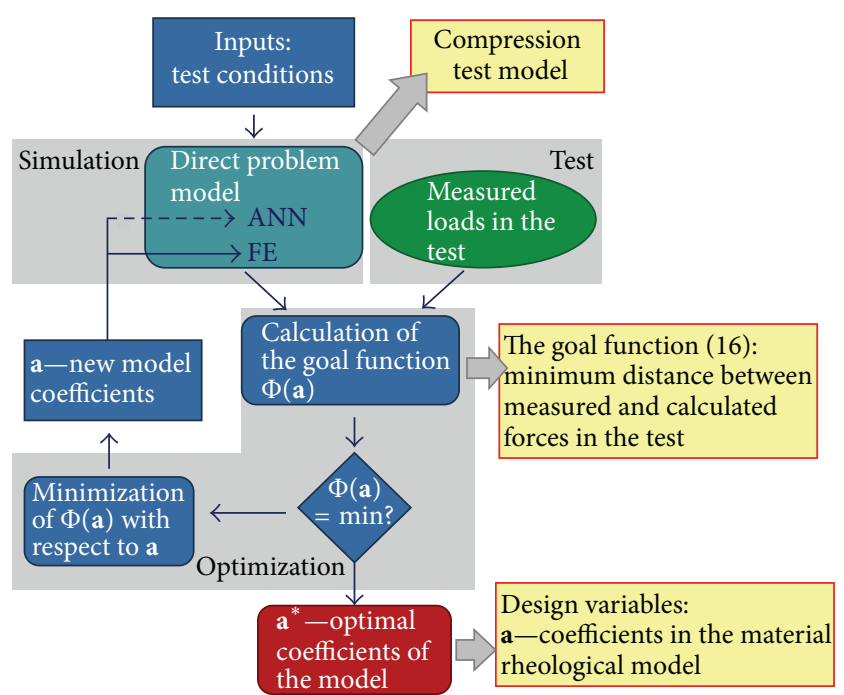

FIGURE 10: Flow chart of the inverse algorithm with the FE model (solid line) and the metamodel (broken line).

was defined as a square root error between measured and calculated loads:

$$
\begin{aligned}
& \Phi(\mathbf{a}, \mathbf{p}) \\
& \quad=\sqrt{\frac{1}{N p t} \sum_{i=1}^{N p t}\left[\frac{1}{N p s} \sum_{j=1}^{N p s}\left(\beta_{i j} \frac{F_{c j i}\left(\mathbf{a}, \mathbf{p}_{i}\right)-F_{m j i}}{F_{m j i}}\right)^{2}\right]},
\end{aligned}
$$

where $F_{m j i}$ and $F_{c j i}$ are measured and calculated loads, $N p t$ is the number of tests, $N p s$ is the number of load measurements in one test, $\mathbf{p}$ is the vector of process parameters (strain rates, temperatures), and $\mathbf{a}$ is the vector of coefficients in the flow stress model. Weights $\beta_{i j}$ in (16) were selected arbitrarily, as explained under (15).

The input of the network included friction coefficient, temperature, and strain rate of the test and coefficients in the 
flow stress model. Since dimensions of samples in plastometric tests are standardized, separate models were developed for each sample dimension. Metamodels for the UC, RC, and PSC tests, which were described in the previous section, were combined with flow stress equations (1), (3), and (4). These metamodels were applied to the identification of the flow stress for materials listed in Section 3. Comparison of the results of the classical inverse analysis with FE direct problem model and the inverse analysis with the metamodel for the investigated materials is given in the following sections.

\subsubsection{Inverse Analysis for the Deformation at Low Temper-} atures. Equation (1) which does not account for material softening due to recrystallization was selected in this part of the analysis. Performed optimization yielded the results given in Table 5. Final values of the objective function (16), which are the measures of the accuracy of the analysis, are given in the last row of this table. It is seen that for steels identification does not show sensitivity of the model to the strain rate and small sensitivity to temperature. Strain rate sensitivity is also small for the $\mathrm{CuCr}$ alloy, but this alloy shows much larger sensitivity to the temperature. Comparison of coefficients in Table 5 with those obtained using conventional inverse analysis with FE model shows that values of coefficients are different, but the final values of the objective function (16) are similar. Beyond copper alloys deformed at temperatures $20-300^{\circ} \mathrm{C}$ (Figure 8), other materials were subjected to the inverse analysis for comparison and good results were obtained as well. It can be concluded that inverse analysis with the metamodel gives accurate results, but uniqueness of identification of (1) is questionable.

5.1.2. Inverse Analysis for Hot Deformation of the Steel DP600. Selected plots of recorded loads during uniaxial hot compression of the DP600 steel are shown in Figure 7. Conventional inverse analysis using algorithm described in [4] was performed for (3) and (4) for the UC tests. The coefficients which were obtained are given in Table 6 . Comparison between the result of the direct inverse analysis [4] and the plots of functions (3) and (4) is shown in Figure 11. The former represents identification of the flow stress model in a tabular form and it is not dependent on the capability of the function to describe behaviour of the material properly. Direct inverse analysis supplies the flow stress model which when substituted to the FE code gives perfect agreement with the measurements of loads. Therefore, it is used as a reference flow stress in this part of the work.

Analysis of the plots in Figure 11 shows that (3) and (4) are not able to describe the flow stress in a large range of temperatures and strain rates; see error $\Phi$ in Table 6, which exceeds $10 \%$. Therefore, equation of [8] and IVM equations (5) and (7) were additionally identified for the DP600 steel. It appeared that these equations are flexible enough to reproduce behaviour of the material properly; see the results for the equation of [8] in Figure 11(c).

The wide range of parameters was used in the present work because the main objective of the research was to investigate inverse with metamodel approach in extreme conditions of identification of the model. It should be emphasized,
TABLE 5: Coefficients in (1) determined for CuCr alloy using inverse analysis with the FE model and with the metamodel.

\begin{tabular}{lcc}
\hline Coeff. & IA + FE & IA + ANN \\
\hline$A$ & 193.2 & 197.6 \\
$B$ & 0.3 & 0.264 \\
$C$ & 0.02 & 0.016 \\
$D$ & 2000.4 & 1975.6 \\
\hline$\Phi$ & 0.0983 & 0.0827 \\
\hline
\end{tabular}

TABle 6: Coefficients in (3) and (4) obtained from the classical inverse analysis for the steel DP600.

\begin{tabular}{lcccccccc}
\hline Equation & $A$ & $B$ & $C$ & $D$ & $E$ & $F$ & $G$ & $\Phi$ \\
\hline$(3)$ & 6038.8 & 0.376 & 0.105 & 0.00337 & 0.521 & - & - & 0.13 \\
$(4)$ & 22.59 & 0.278 & 0.107 & 1589.4 & 0.441 & 6339.5 & 2.956 & 0.124 \\
\hline
\end{tabular}

TAble 7: Coefficients in (3) determined for St3S and CuCr using inverse analysis with the metamodel.

\begin{tabular}{lcc}
\hline Coeff. & St3S & CuCr \\
\hline$A$ & 2622.2 & 1178.4 \\
$B$ & 0.37 & 0.279 \\
$C$ & 0.122 & 0.098 \\
$D$ & 0.0025 & 0.00532 \\
$E$ & 0.727 & 0.624 \\
\hline$\Phi$ & 0.0709 & 0.0888 \\
\hline
\end{tabular}

however, that when the practical range of temperatures is smaller (e.g., $850-1050^{\circ} \mathrm{C}$ for finishing rolling), (4) is accurate (error below $5 \%$ ) and can be efficiently used in modelling.

Capability of the selected material model to reproduce behaviour of the material in the whole range of parameters properly is the main factor, which influences the error of the inverse analysis. To avoid influence of the flexibility of the function on the evaluation of the metamodel, all the results of identification using metamodel will be referred to the results of the classical inverse analysis with FE model of the direct problem.

5.1.3. Identification of the Flow Stress Model (3) Using Inverse Analysis with Metamodel. Performed optimization for (3) and UC tests yielded the results in Table 7. Final values of the objective function (15) are given in the last row of this table. Comparison of coefficients in Table 7 with those obtained using conventional IA + FE approach shows that values of coefficients are different, but the values of the objective function (15) are similar. Beyond this, plots of flow stress as a function of strain obtained from the two inverse approaches are similar; see examples for the $\mathrm{CuCr}$ alloy and alloyed steels in Figure 12. It means that IA with the metamodel gives accurate results, but uniqueness of identification of (3) is questionable. Similar good agreement between the two inverse approaches was obtained for other strain rates and for materials investigated in other projects. 


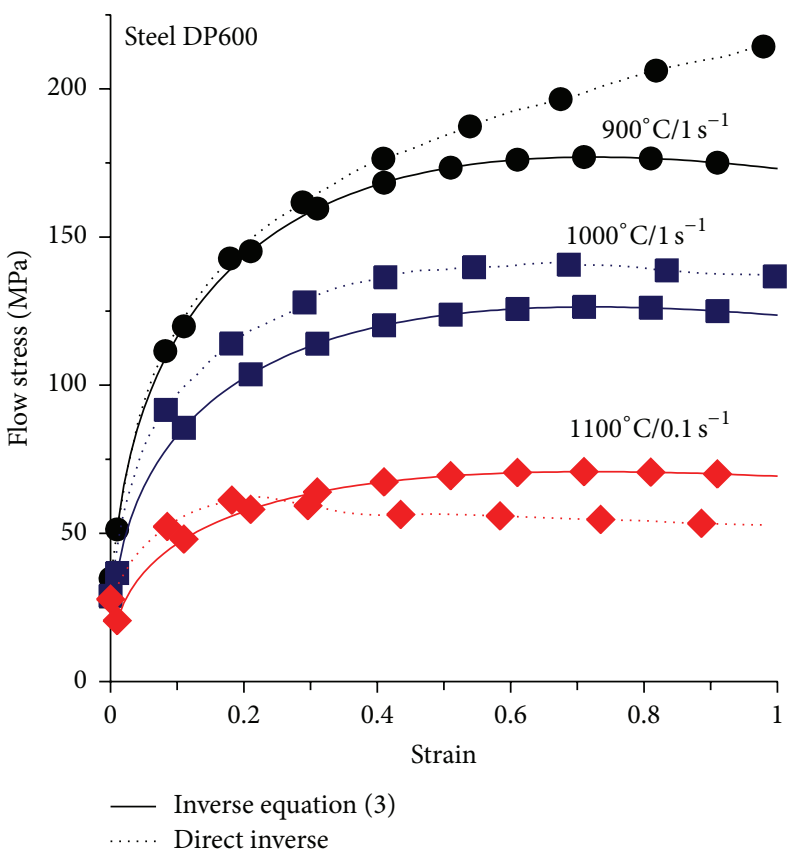

(a)

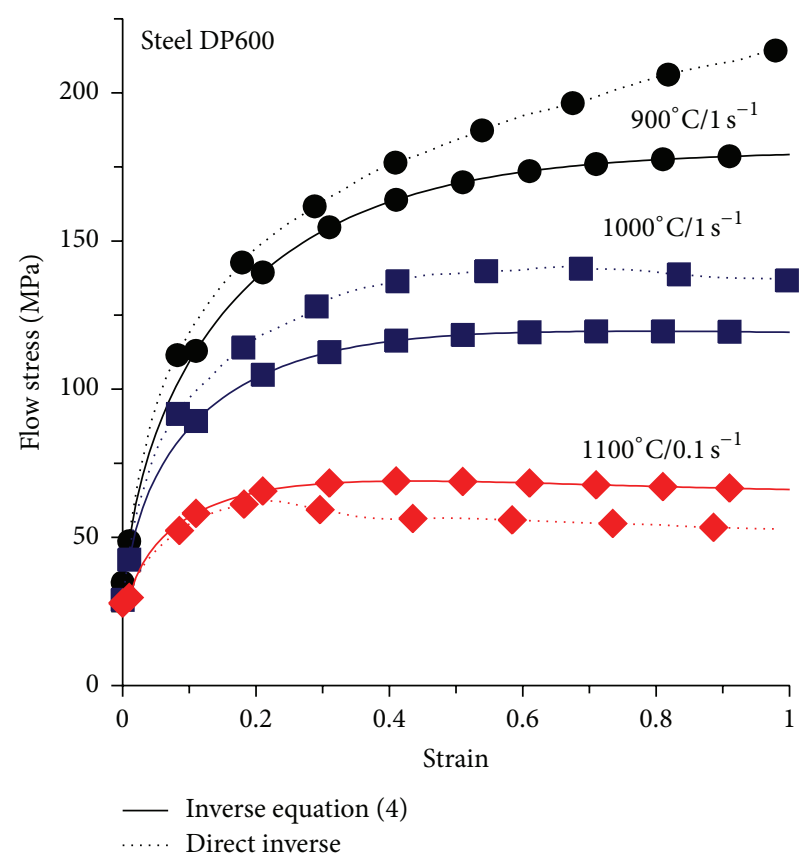

(b)

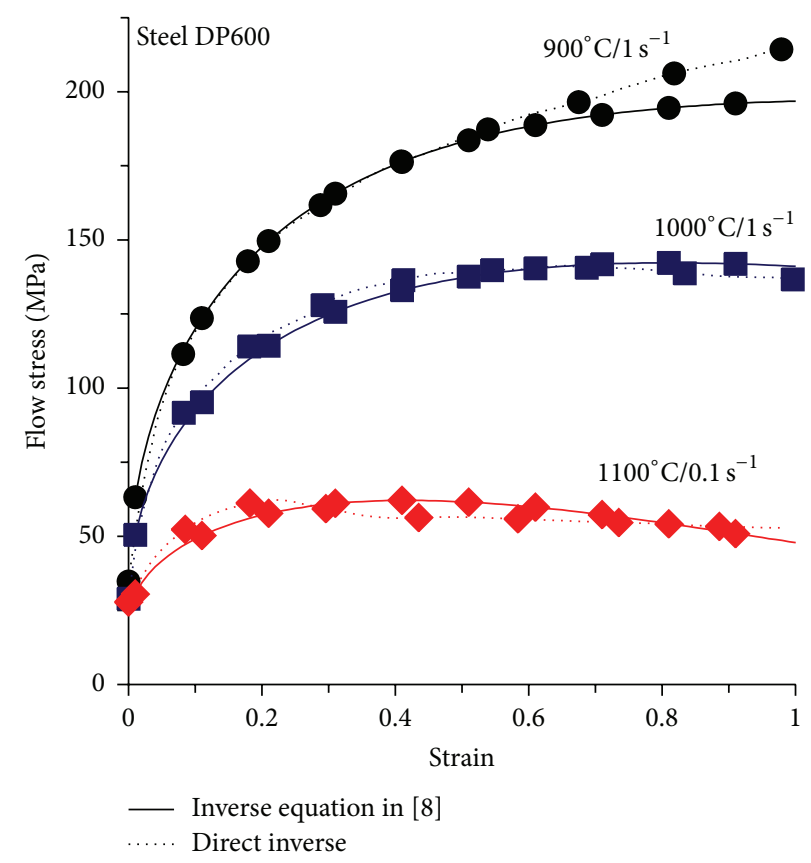

(c)

FIGURE 11: Comparison between the result of the direct inverse analysis (dotted lines) and the plots of functions with coefficients in Table 6 (solid lines): (a) function (3); (b) function (4); (c) model of [8].

5.1.4. Identification of the Flow Stress Model (4) Using Inverse Analysis with Metamodel. This model was also used for hot forming of DP600 steel and performed optimization yielded coefficients $A-G$ in (4). Uniaxial compression tests were considered. Plots of the flow stress as a function of strain obtained from the two inverse approaches did not coincide so well as for (3). It means that inverse analysis with the metamodel was less accurate when (4) with 7 coefficients was identified. It was due to the fact that metamodel itself for (4) was less accurate and these errors of the ANN, although small, were further magnified during optimization. Therefore, clusterization of the network with respect to the value of the force was performed, as proposed in Section 2.3.2. Effectiveness of clusterization method was evaluated by comparison with the conventional inverse approach based on the FE model. Coefficients in (4) calculated using the two methods are given 


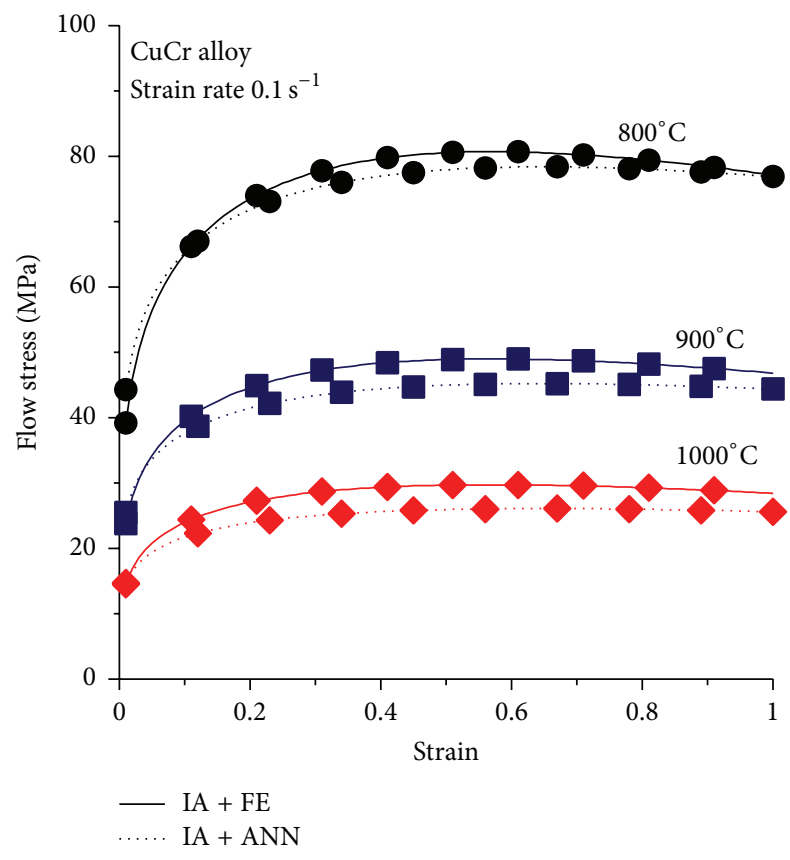

FIGURE 12: Flow stress calculated from (3) with coefficients obtained from the conventional IA + FE approach (solid lines) and inverse analysis with metamodel (dotted lines) for $\mathrm{CuCr}$ alloy, strain rate $0.1 \mathrm{~s}^{-1}$.

in Table 8. Selected plots showing the flow stress calculated for coefficients obtained using the two identification methods are shown in Figure 13. Significant improvement of results was obtained when clusterization of the ANN was applied.

5.1.5. Identification of the Flow Stress Model on the Basis of UC and PSC Tests. Plane state of strains, which is not reachable in other plastometric tests, has inspired for years the scientists to various applications of the PSC tests. Identification of the flow stress model is one of such applications and investigation of the microstructure evolution is another example. Among several research laboratories involved in investigations based on the PSC tests a team led by Sellars at the University of Sheffield should be mentioned. This test was commonly used there for investigation of materials and fundamental works on microstructure evolution [18] and on flow stress models [8] were a result of this research. Application of the FE model of the PSC test to aid the interpretation of results was described in [30] and further analysis of the influence of the size of the sample on the test results was performed in [31]. All these papers showed that PSC tests involve strong inhomogeneities of parameters. In spite of this, due to some specific advantageous features, PSC tests have been widely used for identification of models of various materials like steels [8], aluminium alloys [30, 32], and magnesium alloys [33]. It should be emphasized, however, that large inhomogeneity of deformation, which is caused by complex shape of the deformation zone and by the effect of friction, still makes interpretation of results of PSC tests very difficult. Beyond

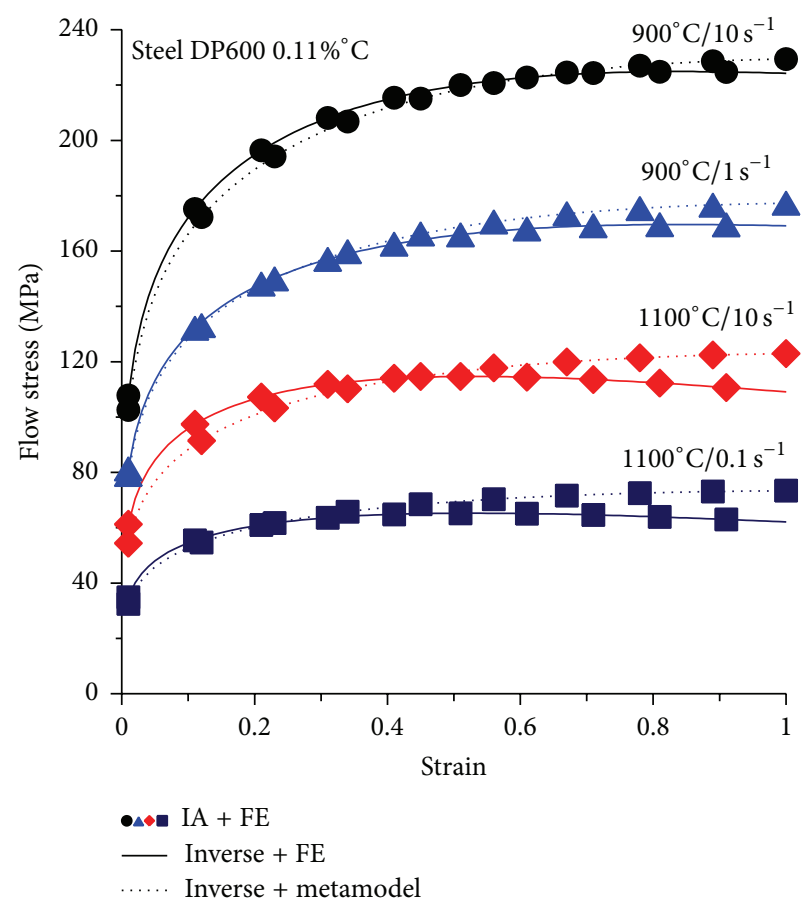

FIGURE 13: Flow stress calculated from (4) with coefficients obtained from the conventional IA + FE approach (filled symbols) and inverse analysis with ANN (broken lines) and with clusterized metamodel (solid lines) for the steel DP600.

this, heat generated due to plastic work and friction, as well as heat transfer to the tools and to the surrounding, causes strong inhomogeneity of the temperature in the sample.

Conventional two-stage inverse analysis [4] for the $\mathrm{CuCr}$ alloy was performed first. The tabular stress-strain data were obtained after the first stage. These data were approximated using (1) or (3) and coefficients obtained in the approximation $\mathbf{a}=\{A, B, C, D\}^{T}$ or $\mathbf{a}=\{A, B, C, D, E\}^{T}$ were used as a starting point for the second stage of the inverse analysis. This point was usually close to the minimum of function (15) and the solution could be obtained in a reasonably short time, in particular when nongradient optimization methods were used. Figure 14 shows stress-strain curves obtained in a tabular form from the first stage of the analysis. It is seen that the results are consistent.

Inverse analysis with the metamodel was performed for both UC and PSC tests. Coefficients in (1) and (3) obtained from this analysis are given in Table 9. Coefficients in (1) and (3) are determined for $\mathrm{CuCr}$ alloy using inverse analysis with the metamodel for the UC and PSC tests. The coefficients were determined by averaging flow curves obtained from the two tests; therefore, the final value of the objection function (15) is not given in this case. This value was 0.0827 and 0.133 for (1) and 0.035 and 0.114 for (3), respectively, for the UC and PSC tests. Analysis of the results shows that conventional inverse analysis gave similar flow curves for both UC and PSC tests; therefore, flow stress determined on the basis of both tests (Table 9) was used in all comparisons in Section 6. 
TABLE 8: Coefficients in (4) determined for DP600 steel using classical inverse analysis with FE model and inverse analysis with the clasterized ANN as metamodel.

\begin{tabular}{lcccccccc}
\hline Method & $A$ & $B$ & $C$ & $D$ & $E$ & $F$ & $G$ \\
\hline IA + FE & 2.423 & 37555 & 0.208 & 0.122 & 0.00519 & 91088 & 0.534 & 0.0754 \\
IA + CANN & 1.509 & 42439 & 0.217 & 0.112 & 3.339 & 7727.5 & 0.211 & 0.0623 \\
\hline
\end{tabular}

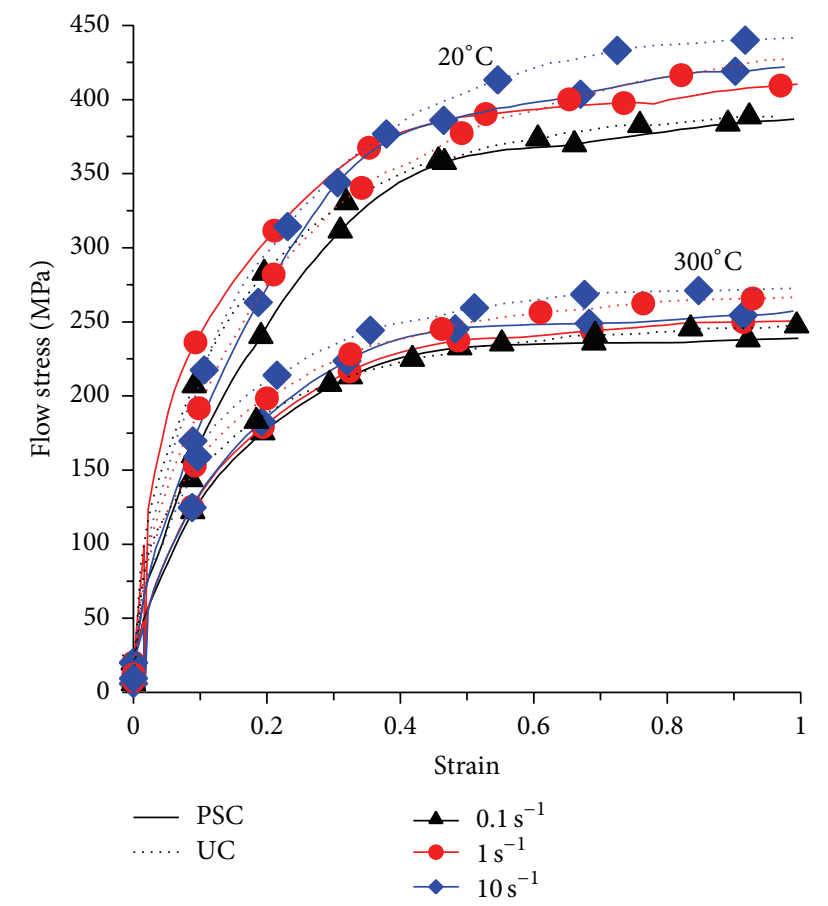

FIGURE 14: Flow stress calculated in a tabular form from the first stage of the inverse analysis.

5.1.6. Identification of the Flow Stress Model on the Basis of $R C$ Tests. Capabilities of the IA with metamodel were further explored for the compression of rings (RC). Various inhomogeneities make the interpretation of results of this test difficult. Due to the fact that ring dimensions after compression are sensitive to friction, this test is frequently used for identification of the friction coefficient [34]. Since determination of the flow stress from the RC test is difficult, researchers usually additionally performed UC tests to identify the flow stress model. Such a procedure is expensive; therefore, inverse analysis with metamodel of the RC test was the next objective of this work. The rings used in tests are standardized; that is, $R=6 \mathrm{~mm}, R=7 \mathrm{~mm}$, or $R=$ $9 \mathrm{~mm}$, and the ratio between $R, r$, and $H$ is $6: 3: 4$ ( $R$ : outer radius, $r$ : inner radius, and $H$ : initial height). The results of identification for the C-Mn and St3S steel with the chemical compositions given in Table 3 are presented below.

ANN-based metamodel of the RC test was built. Coefficients in (3) obtained using classical IA + FE approach and inverse solution with the metamodel are given in Table 10. Final values of the objective function defined by (15) are given in the last column of Table 10. Figure 15 shows flow stress calculated from (4) with coefficients obtained from the RC test using both approaches. Very good agreement between the two methods was obtained.
TABLE 9: Coefficients in (1) and (3) determined for CuCr alloy using inverse analysis with the metamodel for the UC and PSC tests.

\begin{tabular}{lccccc}
\hline Equation & $A$ & $B$ & $C$ & $D$ & $E$ \\
\hline$(1)$ & 201.0 & 0.275 & 0.024 & 1718.6 & - \\
$(3)$ & 735.6 & 0.497 & 0.0201 & 0.0014 & 0.547 \\
\hline
\end{tabular}

TABle 10: Coefficients in (3) determined for the St3S steel using inverse analysis classical with the FE model and with the metamodel of the RC test.

\begin{tabular}{lcccccc}
\hline Method & $A$ & $B$ & $C$ & $D$ & $E$ & $\Phi$ \\
\hline IA + FE & 3694.7 & 0.461 & 1.214 & 0.115 & 0.0276 & 0.1172 \\
IA + ANN & 3036.8 & 0.381 & 0.984 & 0.108 & 0.0271 & 0.0669 \\
\hline
\end{tabular}

Performed research has shown that the number of ANN training data points varied within 1-10 thousand, depending on the considered plastometric test. Training time of the ANN metamodel for various plastometric tests did not exceed 30 minutes (in the case of the largest training data set), while identification with metamodels required 2-10 minutes computing time, depending on the number of identified parameters. One calculation of the objective function using simple FEM model requires 20-30 min. Optimization using simplex method requires about 50-100 calculations of the objective function. Application of more advanced optimization methods inspired by the nature is even more demanding.

5.2. Identification of the Microstructure Evolution Model and the Phase Transformation Model. Equations describing microstructure evolution are simple and the metamodel did not accelerate the inverse analysis. As far as the phase transformation model is considered, two types of neural networks were used. The first was PNN (Probabilistic Neural Network) and the task of this network was to indicate the probability, whether the considered transformation occurs or not. The second type (MLP) was used to predict the starting temperature for the transformations, which were selected by the PNN for the considered conditions; see Figure 16. Modified JMAK model described in [6] was used to generate data for training the metamodels in a wide range of coefficients and cooling rates.

An attempt to apply trained networks to identification coefficients in the phase transformation model was made. Experimental data in the form of dilatometric tests results for the DP600 steel were used. The primary results of optimization were not satisfactory, due to the following problems:

(i) Even small errors in the predictions of the probabilistic network PPN involved large errors in the identification of coefficients. This aspect is the objective of 


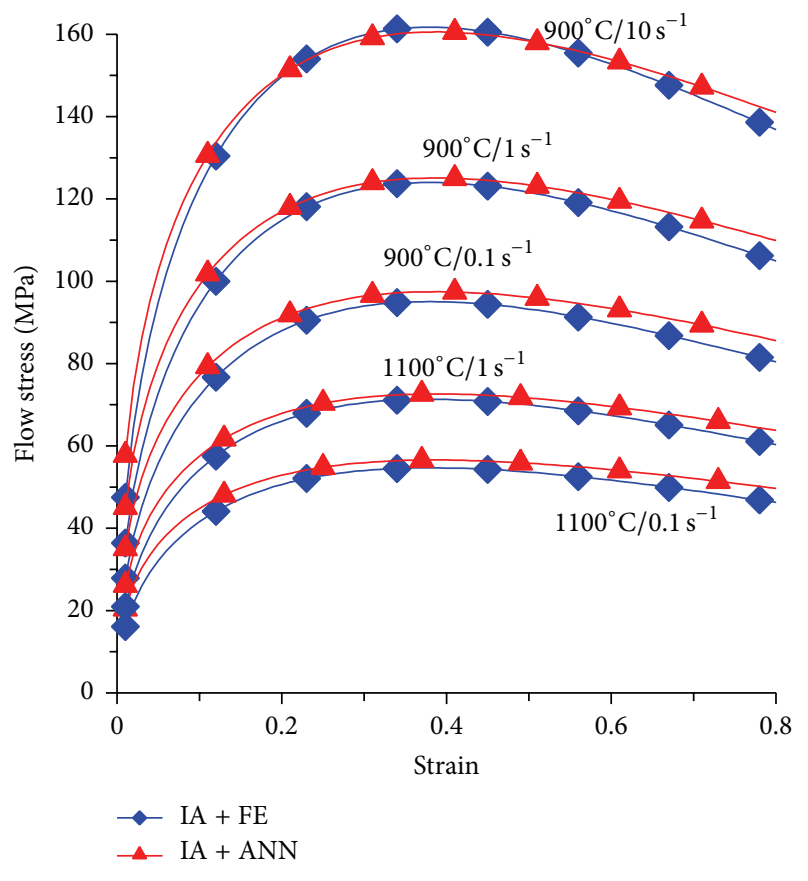

FIGURE 15: Flow stress calculated from (3) with coefficients obtained from the RC test using the conventional inverse analysis with the FE model and inverse analysis with the metamodel.

further research and possibilities of improvement of classification will be searched for.

(ii) The networks predicted kinetics of transformation (transient process) for given boundary conditions, which were determined from the equilibrium diagram (Thermo-Calc software was used). It means that trained networks could be used for steels with similar phase equilibrium diagram, which made inverse analysis less efficient.

Recapitulating this part of the research, it was concluded that application of the metamodel does not improve efficiency of the inverse analysis in the case of the phase transformation model. Therefore, conventional inverse solution based on the simplex optimization method was used in the present work.

\section{Validation}

Performed analysis has shown that, among models considered in this work, application of metamodels in the identification procedure was efficient for the flow stress models only. The decrease of the computing costs in the case of the microstructure evolution model and phase transformation model was negligible. Therefore, flow stress models were validated by comparing the results qualitatively and quantitatively with the experimental data. Comparison between forces measured in the tests and calculated by the FE model, with one of (1)-(5) with optimal coefficients used in the constitutive law, was the basis of the validation.
6.1. Flow Stress Determined from the UC Test. Loads predicted by the FE code with flow stress calculated from (1) with coefficients in Table 5 are compared with measurements for the $\mathrm{CuCr}$ alloy in Figure 17. To demonstrate the universality of the method, the results for the two steels are shown in this figure as well. Very good accuracy of the inverse solution with metamodel for (1) for low deformation temperatures was confirmed.

Observations made in Section 5.1.2 were confirmed in Figure 18, where comparison between loads measured in the hot tests and calculated by the FE code with flow stress equations (3) and (4) with coefficients determined using conventional inverse analysis is shown. As a reference, results for the equation of [8] (published also in [30,31]) are shown in this figure. Again some discrepancies were obtained for (3) and (4), while a very good agreement between calculations and measurements was obtained for the equation of [8].

Analysis of the results presented in Section 5.1.3 shows good agreement between flow stress calculated from (3) with coefficients determined by the inverse analysis with the FE model and inverse analysis with metamodel. To verify these results, loads predicted by the FE code with flow stress given by (3) with coefficients in Table 7 were compared with measurements for all the investigated materials. The selected results of this comparison are shown in Figure 19. Reasonably good agreement was obtained for all materials and all conditions of deformation. Some discrepancies, which were observed, were definitely due to the lack of capability of (3) to describe behaviour of investigated materials in a wide range of temperatures and strain rates properly. It can be concluded that the inverse analysis with metamodel gives results, which are very close to those obtained from the classical inverse analysis with the FE model, while the former approach is few orders of magnitude faster.

Loads predicted by the FE code with flow stress calculated from (4) with coefficients in Table 8 were compared with measurements for all the investigated steels. Selected results of this comparison for the DP steel containing $0.11 \%{ }^{\circ} \mathrm{C}$ are shown in Figure 20. In calculations, three methods of identification of coefficients in (4) were used: classical inverse with $\mathrm{FE}$ direct problem model, inverse with one ANN metamodel, and inverse with clusterized network metamodel. It is seen in this figure that in general very good agreement between various results was obtained. Application of clusterized network in the inverse analysis improved the agreement with the classical inverse analysis.

6.2. Flow Stress Determined from Various Tests. This part of verification of the models was performed by comparison between forces measured in various tests and calculated by the FE code with the identified model introduced in the constitutive law. Identification of the models was performed on the basis of the UC and PSC tests. Figure 21 shows comparison of forces measured in both tests and calculated by the FE code with (1) with coefficients obtained from the inverse analysis with the metamodel for both UC and PSC tests (Table 9). Similar comparison for (3) is shown in Figure 22. 


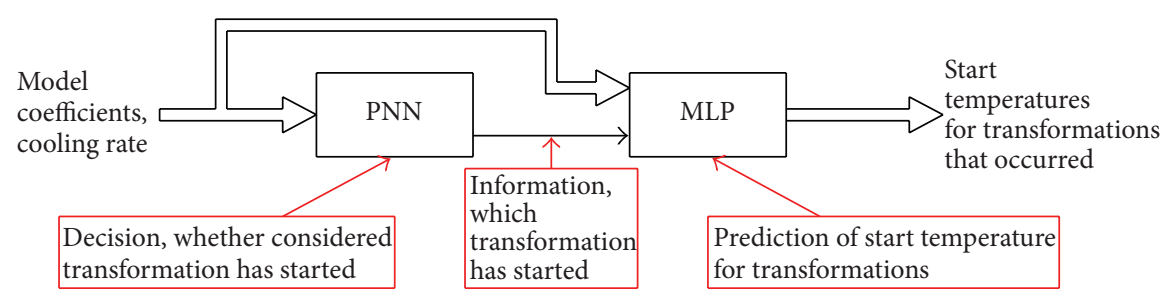

FIGURE 16: The general idea of using PNN and MLP as phase transformations metamodel.

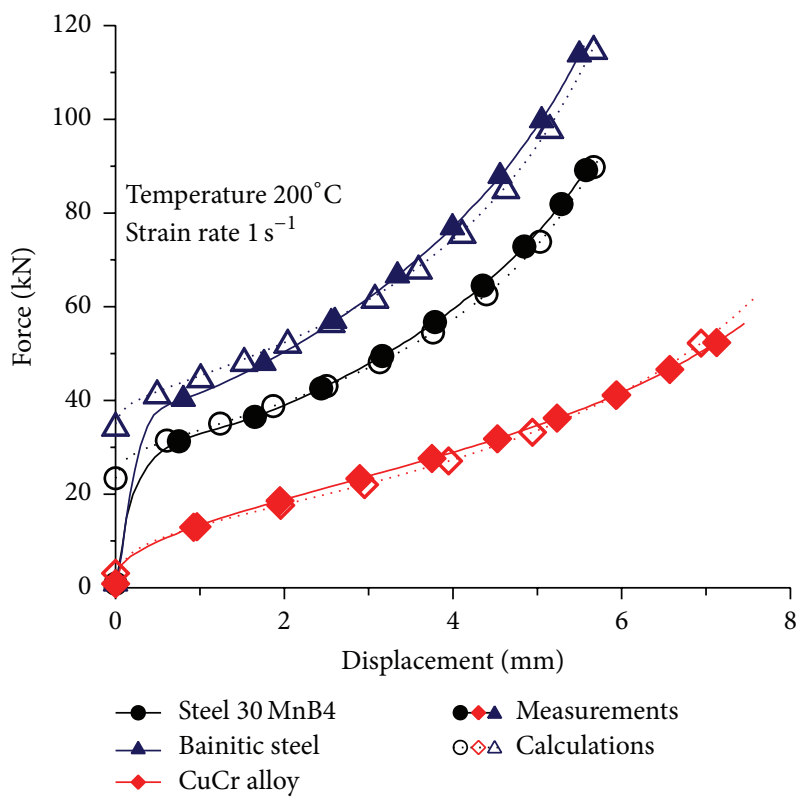

FIGURE 17: Loads predicted by the FE code with flow stress calculated from (1) with coefficients determined using inverse analysis with metamodel compared with the measurements for the $\mathrm{CuCr}$ alloy and for the two steels.

It is seen in Figures 21 and 22 that (1) is not able to describe behaviour of the material in the plane strain compression test in the investigated range of temperatures and strain rates, when identification was performed on the basis of the two tests. Much better results were obtained for (3).

Figure 23 shows comparison of forces measured in the RC tests and calculated by the FE code with (3) with coefficients obtained from the inverse analysis with the metamodel for this test (Table 10).

Possibility of identification of the flow stress model on the basis of the inverse analysis with metamodel for the PSC and RC tests was confirmed, although it is more difficult and time consuming than the UC tests. Conventional inverse analysis performed for the uniaxial compression and plane strain compression gave similar flow stress model. The model determined on the basis of both tests gave very good prediction of forces.

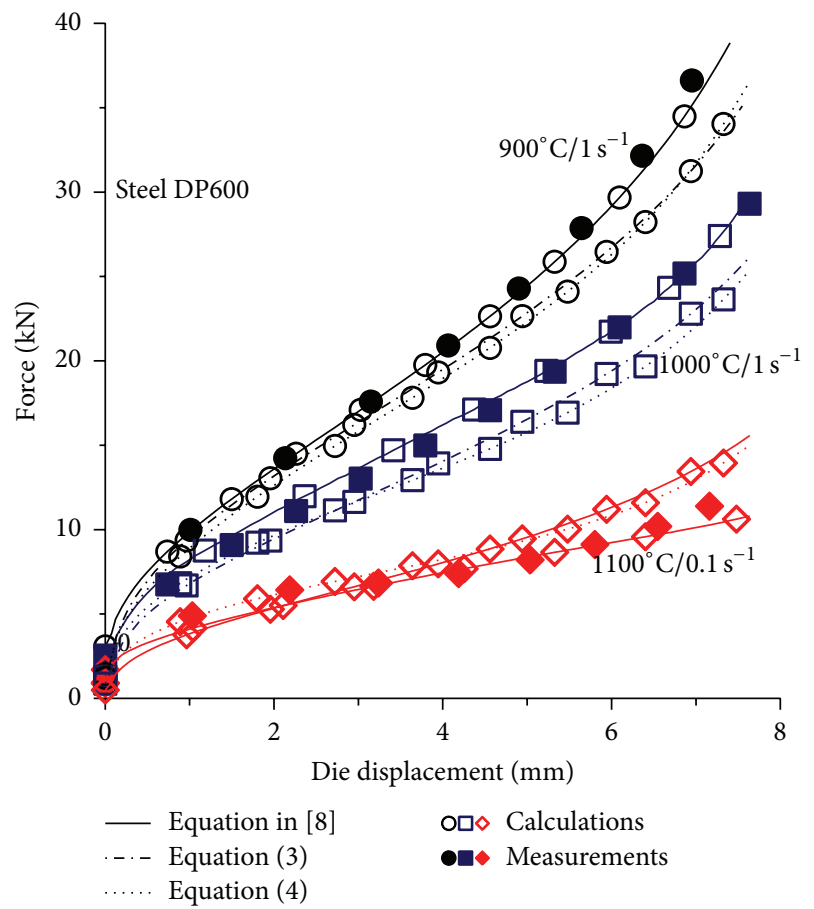

FIGURE 18: Comparison between loads measured in the tests (filled symbols) and calculated by the FE code with flow stress equations (3) and (4) and equation of [8] with coefficients determined using conventional inverse analysis (open symbols).

\section{Discussion of Results and Conclusions}

The general conclusion from the performed research is that the accuracy of the inverse solution (final value of the objective function) depends on two factors:

(1) capability of the selected material model to reproduce behaviour of the material in the whole range of parameters properly;

(2) accuracy of the optimization methods and capability to avoid local minima.

Possibility of application of metamodels in the inverse analysis was confirmed. Metamodels for various tests and for various models were developed and applied in the inverse analysis. Significant decrease of the computing time was obtained when FE model was substituted by metamodel. 


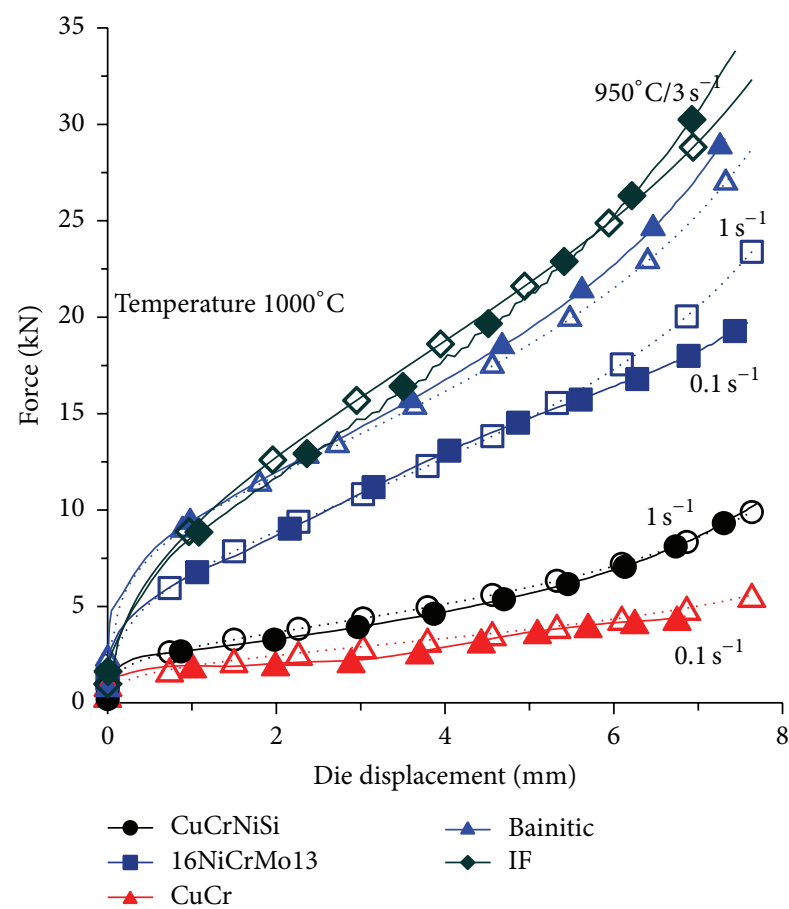

FIGURE 19: Selected examples of comparison of the loads measured in the tests (solid lines) and calculated by the FE code (dotted lines) with flow stress model (3) with coefficients determined by the inverse analysis with metamodel.

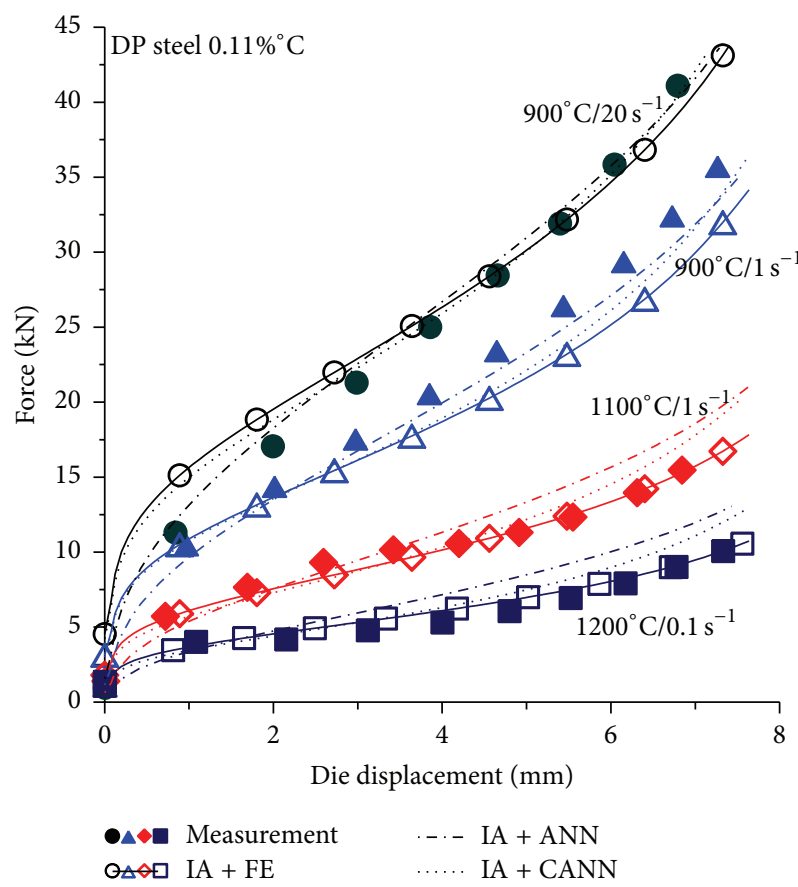

FIGURE 20: Loads measured in the tests (full symbols) and predicted by the FE code with flow stress calculated from (4) with coefficients determined using classical inverse analysis with the FE model (solid lines) and the two metamodels (ANN and CANN).
Results of identification using metamodel are in good agreement with the classical inverse analysis with FE model of the direct problem. The general observations from this part of the work are as follows:

(i) Metamodels are efficient in applications to identification of coefficients in flow stress models on the basis of various experimental tests. Inverse analysis with the metamodel is few orders of magnitude faster than the conventional approach with the FE model; see Section 5. Accuracy of the former approach is quite good and it should be recommended for practical applications of the inverse analysis.

(ii) Long computing times are needed for training the metamodel, but once trained the metamodel can be applied to any new material, assuming that the dimensions of the sample and the flow stress equation do not change.

(iii) Although the values of coefficients obtained from various tests may differ, the agreement between measured and calculated forces is good. It means that there is no unique solution of the problem, but the accuracy of the obtained solution is satisfactory.

(iv) The general observation in the paper was that good accuracy of the training of the metamodel (ANN) was obtained for all experiments and for all material models. However, when combined with the optimization in the inverse analysis the performance of the metamodel was decreasing with increasing number of coefficients in the model. Thus, good accuracy of the IA with the metamodel was obtained for (1) and (3) with 4 and 5 coefficients, respectively. In the case of (4) with 7 coefficients, identification with conventional ANN metamodel did not give good accuracy, but after clusterization this good accuracy was obtained.

(v) Validation of the solution with the metamodel by comparison of measured and calculated compression forces confirmed its good accuracy for models with lower numbers of coefficients. Increase of number of coefficients resulted in a decrease of the accuracy.

(vi) Attempts to develop metamodel for the compression tests combined with equation of [8] or with the IVM equation (5) were not successful. These models give very good description of the material response to deformation, in particular when dynamic recrystallization is involved, but they require time consuming identification procedure using classical inverse with the FE direct problem model.

(vii) Generally all investigated flow stress models describe properly materials response for lower values of the Zener-Hollomon parameter $Z$ (higher temperatures, lower strain rates). Larger discrepancies occur for higher $Z$.

(viii) An attempt to apply metamodelling to identification of microstructure evolution model was not successful. Identification based on the stress relaxation tests 


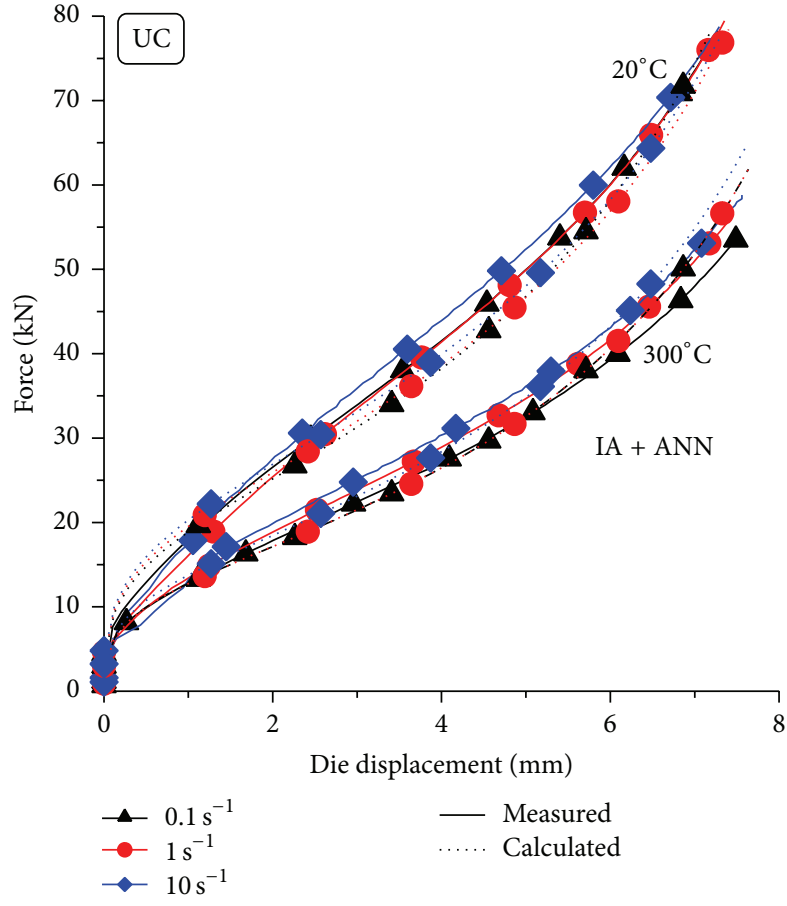

(a)

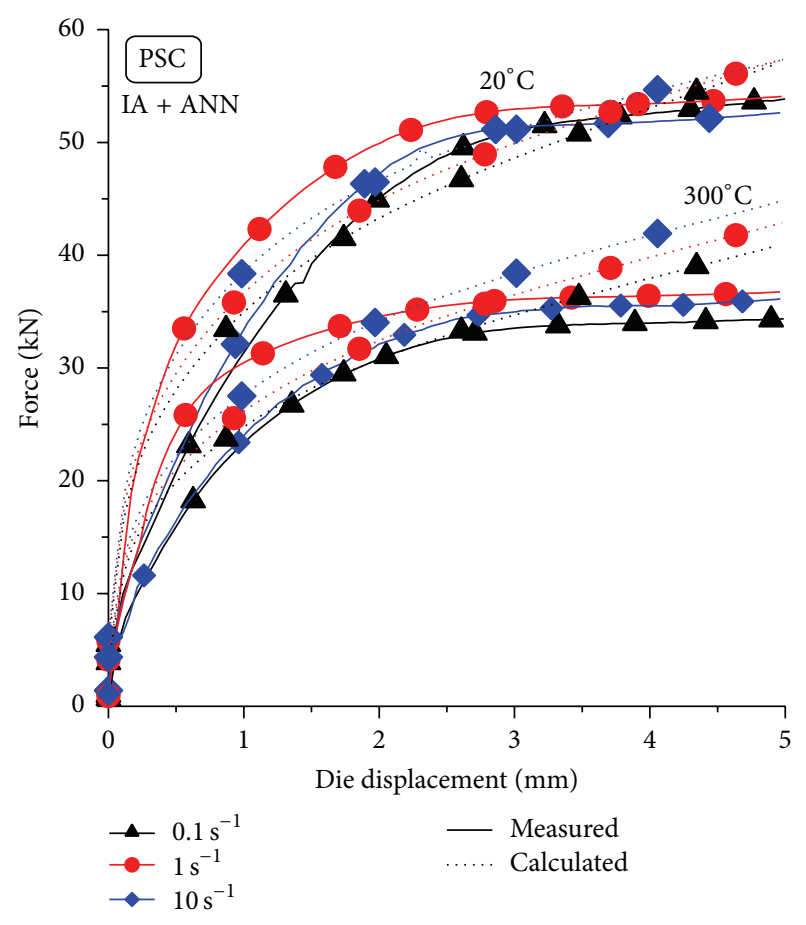

(b)

FIGURE 21: Comparison of forces measured in the uniaxial compression (a) and plane strain compression (b) and calculated by the FE code with (1) with the coefficients obtained from the inverse analysis with the metamodel for both UC and PSC tests (row 1 in Table 9).

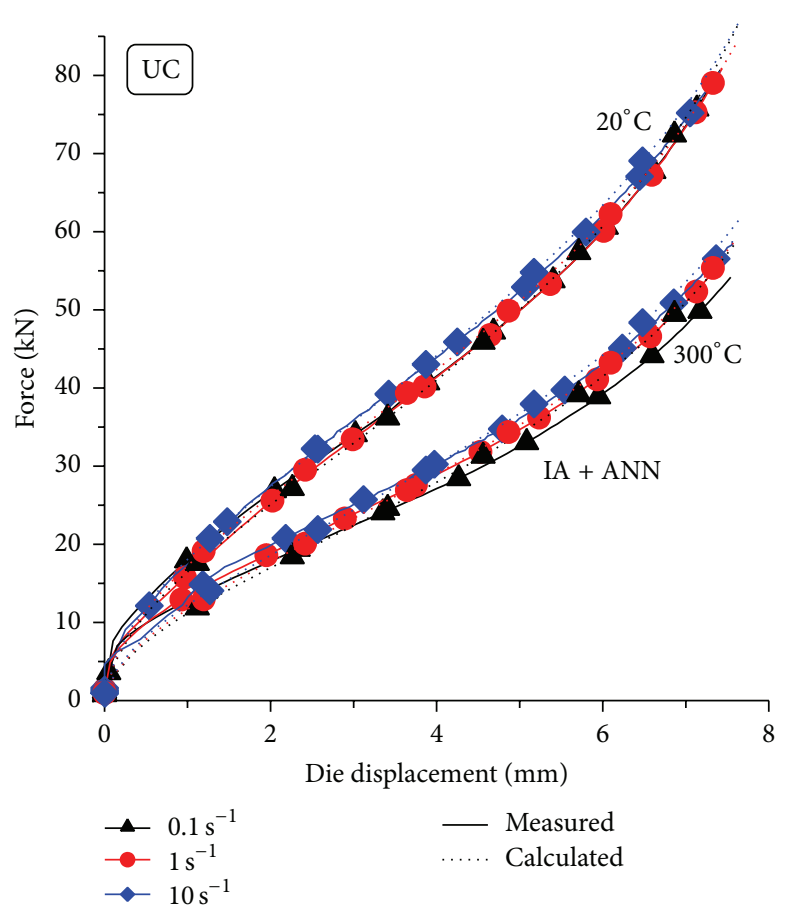

(a)

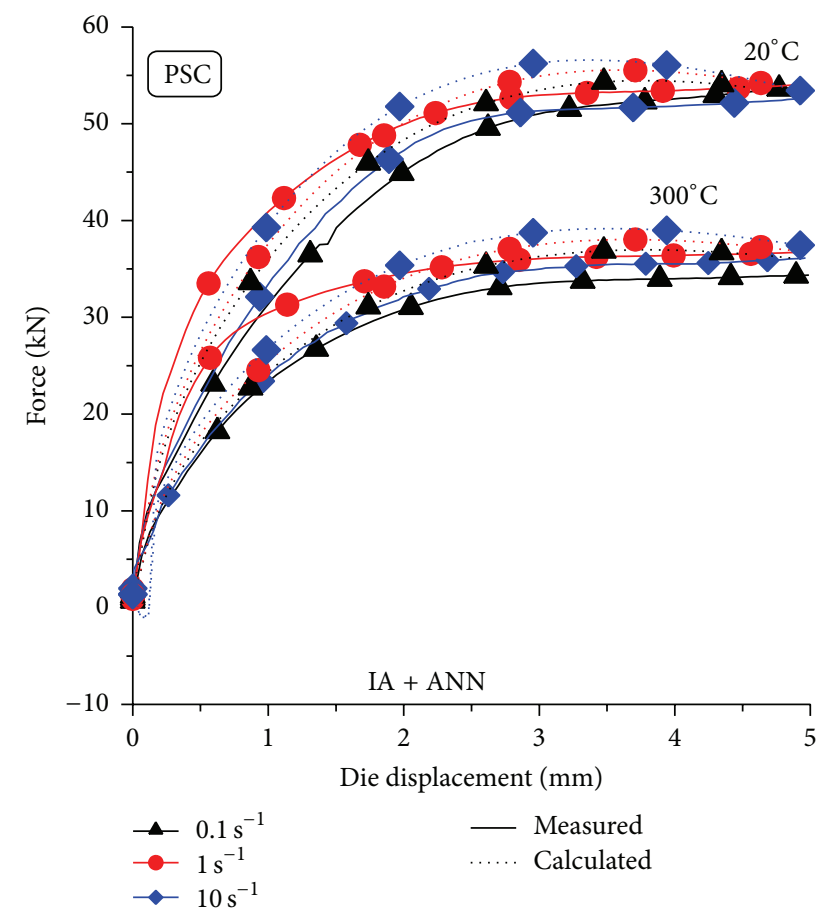

(b)

FIGURE 22: Comparison of forces measured in the UC (a) and PSC (b) tests and calculated by the FE code with (3) with the coefficients obtained from the inverse analysis with the metamodel for both of these tests (row 2 in Table 9). 


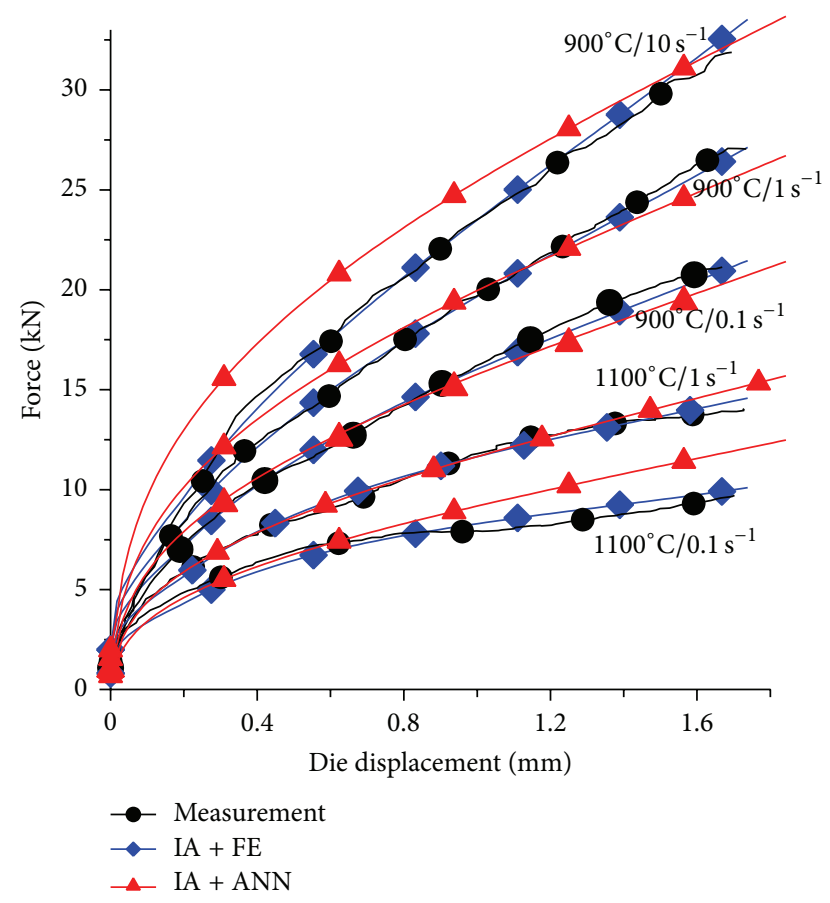

FIGURE 23: Comparison of forces measured in the ring compression tests for the St3S steel and calculated by the FE code with (3) with the coefficients obtained from the inverse analysis with the metamodel.

does not require long FE calculations; therefore, an advantage from using the metamodel was negligible.

(ix) Application of metamodelling to identification of phase transformation model showed that this task requires two metamodels, PPN and MPL networks. The first gives information whether a considered transformation occurs and the second gives information about transformation temperature. Numerical tests showed that this two-network approach gives reasonably good predictions of transformation temperatures, but it was not successful in combination with the optimization methods in the inverse analysis.

\section{Conflict of Interests}

The authors declare that there is no conflict of interests regarding the publication of this paper.

\section{Acknowledgment}

The authors acknowledge the financial assistance of the NCN, Project no. N508 629740.

\section{References}

[1] J. C. Gelin and O. Ghouati, "An inverse method for determining viscoplastic properties of aluminium alloys," Journal of Materials Processing Technology, vol. 45, no. 1-4, pp. 435-440, 1994.

[2] A. Gavrus, E. Massoni, and J. L. Chenot, "An inverse analysis using a finite element model for identification of rheological parameters," Journal of Materials Processing Technology, vol. 60, no. 1-4, pp. 447-454, 1996.

[3] S. Khoddam, Y. C. Lam, and P. F. Thomson, "Numerical results achieved with an inverse computational method for determining the constitutive parameters using the hot torsion test results," Steel Research, vol. 67, no. 2, pp. 39-43, 1996.

[4] D. Szeliga, J. Gawąd, and M. Pietrzyk, "Inverse analysis for identification of rheological and friction models in metal forming," Computer Methods in Applied Mechanics and Engineering, vol. 195, no. 48-49, pp. 6778-6798, 2006.

[5] L. P. Karjalainen and J. Perttula, "Characteristics of static and metadynamic recrystallization and strain accumulation in hotdeformed austenite as revealed by the stress relaxation method," ISIJ International, vol. 36, no. 6, pp. 729-736, 1996.

[6] M. Pietrzyk and R. Kuziak, "Modelling phase transformations in steel," in Microstructure Evolution in Metal Forming Processes, J. Lin, D. Balint, and M. Pietrzyk, Eds., pp. 145-179, Woodhead Publishing, Oxford, UK, 2012.

[7] Ł. Sztangret, D. Szeliga, J. Kusiak, and M. Pietrzyk, "Application of inverse analysis with metamodelling for identification of metal flow stress," Canadian Metallurgical Quarterly, vol. 51, no. 4, pp. 440-446, 2012.

[8] S. B. Davenport, N. J. Silk, C. N. Sparks, and C. M. Sellars, "Development of constitutive equations for modelling of hot rolling," Materials Science and Technology, vol. 16, no. 5, pp. 539$546,2000$.

[9] R. Rikards and J. Auzins, "Response surface method for solution of structural identification problems," Inverse Problems in Science and Engineering, vol. 12, no. 1, pp. 59-70, 2004.

[10] A. C. Rutherford, D. J. Inman, G. Park, and F. M. Hemez, "Use of response surface metamodels for identification of stiffness and damping coefficients in a simple dynamic system," Shock and Vibration, vol. 12, no. 5, pp. 317-331, 2005.

[11] S. E. Fang and R. Perera, "A metamodel based damage identification method," Advanced Materials Research, vol. 163-167, pp. 2704-2708, 2010.

[12] L. W. Meyer, A. Weise, and F. Hahn, "Comparison of constitutive flow curve relations in cold and hot forming," Journal of Physics IV France, vol. 7, no. C3, pp. C3-13-C3-20, 1997.

[13] F. Grosman, "Application of a flow stress function in programmes for computer simulation of plastic working processes," Journal of Materials Processing Technology, vol. 64, no. 1-3, pp. 169-180, 1997.

[14] H. Mecking and U. F. Kocks, "Kinetics of flow and strainhardening," Acta Metallurgica, vol. 29, no. 11, pp. 1865-1875, 1981.

[15] Y. Estrin and H. Mecking, "A unified phenomenological description of work hardening and creep based on oneparameter models," Acta Metallurgica, vol. 32, no. 1, pp. 57-70, 1984.

[16] R. Sandström and R. Lagneborg, "A model for hot working occurring by recrystallization," Acta Metallurgica, vol. 23, no. 3, pp. 387-398, 1975.

[17] A. Hensel and T. Spittel, Kraft-und Arbeitsbedarf Bildsamer Formgebungsverfahren, VEB Deutscher Verlagfür Grundstoffindustrie, Leipzig, Germany, 1978.

[18] C. M. Sellars, "Physical metallurgy of hot working," in Hot Working and Forming Processes, C. M. Sellars and G. J. Davies, Eds., pp. 3-15, The Metals Society, London, UK, 1979.

[19] J. Ordon, R. Kuziak, and M. Pietrzyk, "History dependent constitutive law for austenitic steels," in Proceedings of the Metal Forming 2000, M. Pietrzyk, J. Kusiak, J. Majta, P. Hartley, and I. Pillinger, Eds., pp. 747-753, A. Balkema, Kraków, Poland, 2000. 
[20] J. Kusiak, Ł. Sztangret, Ł. Rauch, and M. Pietrzyk, "Metamodel driven optimization of thermomechanical industrial processes," Computer Methods in Materials Science, vol. 14, no. 1, pp. 20-26, 2014.

[21] L. Fausett, Fundamentals of Neural Networks: Architectures, Algorithms, and Applications, Prentice-Hall, Upper Saddle River, NJ, USA, 1994.

[22] M. L. Stein, Interpolation of Spatial Data: Some Theory for Kriging, Springer, New York, NY, USA, 1999.

[23] W. C. M. Van Beers and J. P. C. Kleijnen, "Kriging interpolation in simulation: a survey," in Proceedings of the Winter Simulation Conference, R. G. Ingalls, M. D. Rossetti, J. S. Smith, and B. A. Peters, Eds., vol. 1, pp. 113-121, Washington, DC, USA, December 2004.

[24] J. Kusiak, Ł. Sztangret, and M. Pietrzyk, "Effective strategies of metamodelling of industrial metallurgical processes," Advances in Engineering Software, vol. 89, pp. 90-97, 2015.

[25] J. G. Lenard, M. Pietrzyk, and L. Cser, Mathematical and Physical Simulation of the Properties of Hot Rolled Products, Elsevier, Amsterdam, The Netherlands, 1999.

[26] M. Kleiber, H. Antunez, T. D. Hien, and P. Kowalczyk, Parameter Sensitivity in Nonlinear Mechanics, Wiley, 1997.

[27] M. D. Morris, "Factorial sampling plans for preliminary computational experiments," Technometrics, vol. 33, no. 2, pp. 161-174, 1991.

[28] D. Szeliga, Metal Forming Identification Problems-Comprehensive Study, AGH University of Science and Technology Press, Kraków, Poland, 2013.

[29] D. Szeliga and M. Pietrzyk, "Identification of rheological models and boundary conditions in metal forming," International Journal of Materials and Product Technology, vol. 39, no. 3-4, pp. 388-405, 2010.

[30] B. Kowalski, C. M. Sellars, and M. Pietrzyk, "Development of a computer code for the interpretation of results of hot plane strain compression tests," ISIJ International, vol. 40, no. 12, pp. 1230-1236, 2000.

[31] B. Kowalski, C. M. Sellars, and M. Pietrzyk, "Identification of rheological parameters on the basis of plane strain compression tests on specimens of various initial dimensions," Computational Materials Science, vol. 35, no. 2, pp. 92-97, 2006.

[32] N. J. Silk and M. R. van der Winden, "Interpretation of hot plane strain compression testing of aluminium specimens," Materials Science and Technology, vol. 15, no. 3, pp. 295-300, 1999.

[33] M. D. Nave and M. R. Barnett, "Microstructures and textures of pure magnesium deformed in plane-strain compression," Scripta Materialia, vol. 51, no. 9, pp. 881-885, 2004.

[34] J. A. Schey, "Friction effects in metalworking processes," in Metal Deformation Processes: Friction and Lubrication, J. A. Schey, Ed., pp. 17-81, Marcel Dekker, New York, NY, USA, 1970. 

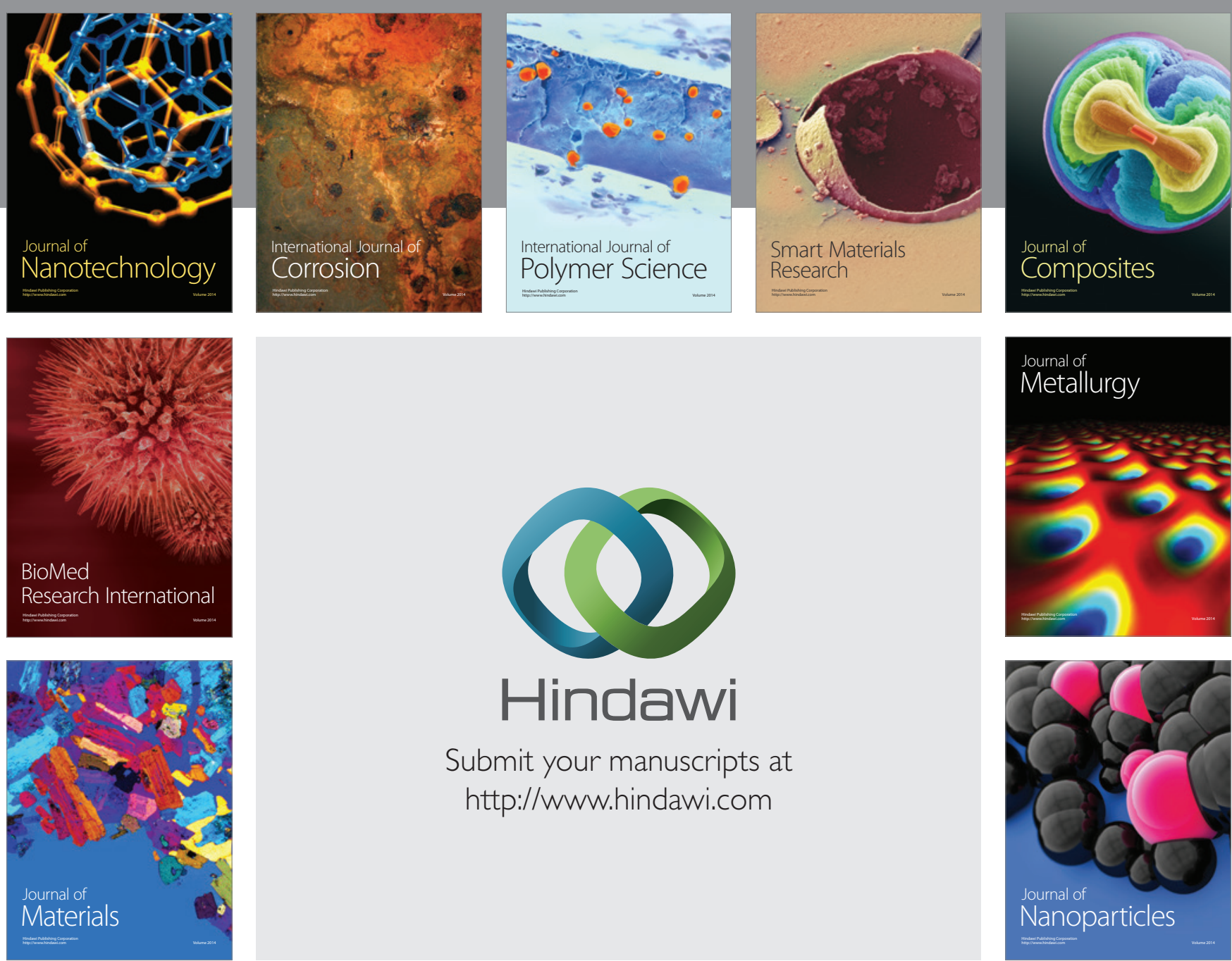

\section{Hindawi}

Submit your manuscripts at

http://www.hindawi.com

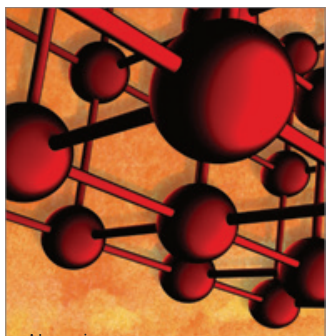

Materials Science and Engineering
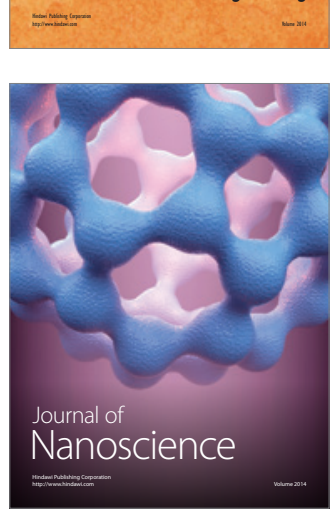
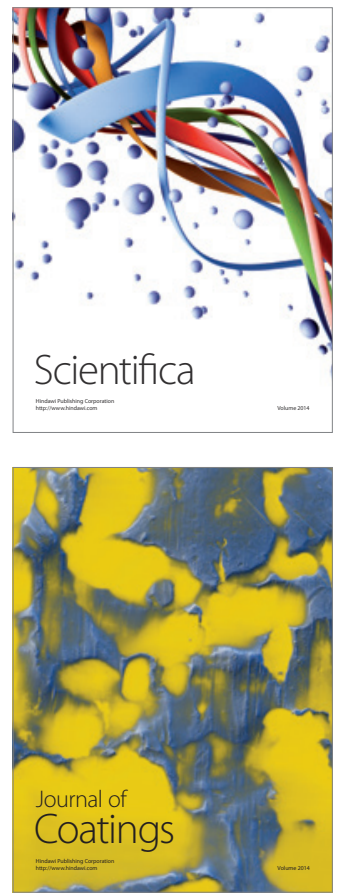
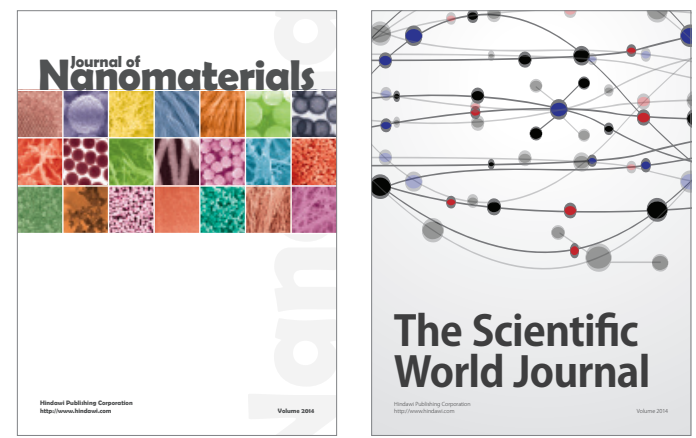

The Scientific World Journal
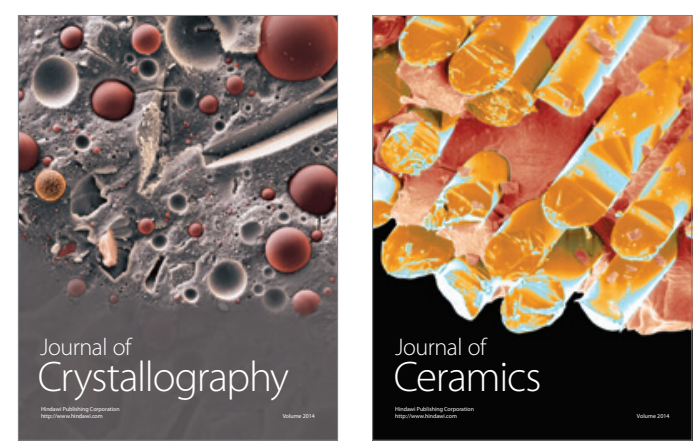
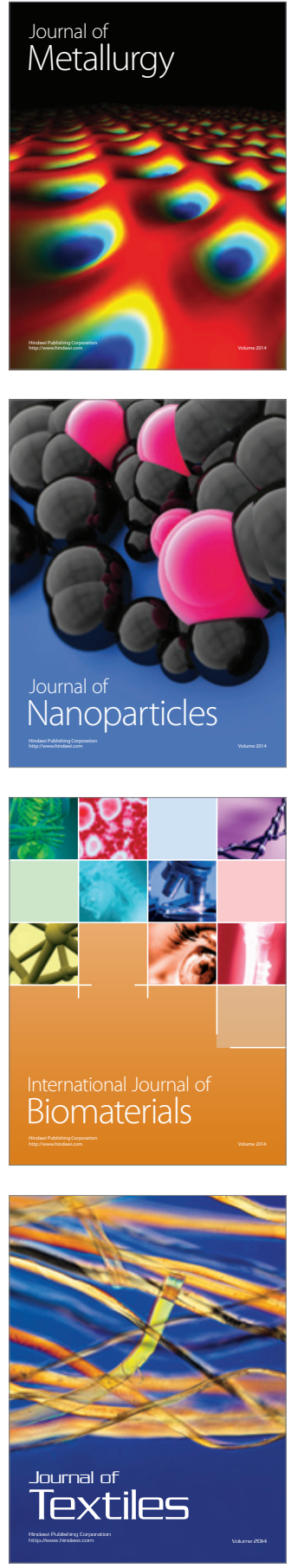